\title{
PML-RARA requires DNA methyltransferase 3A to initiate acute promyelocytic leukemia
}

\author{
Christopher B. Cole, ${ }^{1}$ Angela M. Verdoni, ${ }^{1}$ Shamika Ketkar, ${ }^{1}$ Elizabeth R. Leight, ${ }^{1}$ David A. Russler-Germain, ${ }^{1}$ \\ Tamara L. Lamprecht, ${ }^{1}$ Ryan T. Demeter, ${ }^{2}$ Vincent Magrini, ${ }^{2}$ and Timothy J. Ley ${ }^{1,2}$ \\ 'Departments of Medicine and Genetics, Division of Oncology, Section of Stem Cell Biology, and ${ }^{2}$ The McDonnell Genome Institute, Washington University School of Medicine, St. Louis, Missouri, USA.
}

\begin{abstract}
The DNA methyltransferases DNMT3A and DNMT3B are primarily responsible for de novo methylation of specific cytosine residues in $\mathrm{CpC}$ dinucleotides during mammalian development. While loss-of-function mutations in DNMT3A are highly recurrent in acute myeloid leukemia (AML), DNMT3A mutations are almost never found in AML patients with translocations that create oncogenic fusion genes such as PML-RARA, RUNX1-RUNX1T1, and MLL-AF9. Here, we explored how DNMT3A is involved in the function of these fusion genes. We used retroviral vectors to express PML-RARA, RUNX1-RUNX1T1, or MLL-AF9 in bone marrow cells derived from WT or DNMT3A-deficient mice. Additionally, we examined the phenotypes of hematopoietic cells from Ctsg-PML-RARA mice, which express PML-RARA in early hematopoietic progenitors and myeloid precursors, with or without DNMT3A. We determined that the methyltransferase activity of DNMT3A, but not DNMT3B, is required for aberrant $P M L-R A R A$-driven self-renewal ex vivo and that DNMT3A is dispensable for RUNX1-RUNX1T1- and MLL-AF9-driven self-renewal. Furthermore, both the $P M L-R A R A$-driven competitive transplantation advantage and development of acute promyelocytic leukemia (APL) required DNMT3A. Together, these findings suggest that PML-RARA requires DNMT3A to initiate APL in mice.
\end{abstract}

\section{Introduction}

Recent efforts by our group and others have identified most of the highly recurrent somatic mutations that are relevant for the pathogenesis of acute myeloid leukemia (AML) (1). In addition to discovering recurrent mutations in genes that were not previously known to be important for AML, patterns of mutational cooccurrence and mutual exclusivity are providing important clues regarding the biology of pathways that may contribute to this disease. Mutations in DNMT3A, one of the two mammalian de novo DNA methyltransferases, occur in approximately $20 \%$ of patients with AML; however, they almost never co-occur with the common chromosomal translocations that create fusion genes such as $P M L$ RARA and RUNX1-RUNX1T1 (also referred to as AML-ETO) and $M L L$ fusion genes such as $M L L-A F 9(1,2)$, suggesting a possible relationship between these fusion oncogenes and a fully functional DNMT3A to initiate leukemia.

DNMT3A and the highly homologous enzyme DNMT3B are responsible for inducing specific patterns of de novo DNA methylation in bone marrow (BM) stem/progenitor cells, which is important for the ability of hematopoietic stem cells (HSCs) to develop into differentiated peripheral blood (PB) cells (3-5). The most common DNMT3A mutation, which leads to a heterozygous $\mathrm{R} 882 \mathrm{H}$ amino acid change in the catalytic domain of the enzyme, reduces DNMT3A methylase activity by $80 \%$ and, in a dominant-negative fashion, inhibits the ability of WT DNMT3A protein to form active homotetramers $(6,7)$. Although a requirement for functional DNMT3A has not been tested for any of the common

Conflict of interest: The authors have declared that no conflict of interest exists Submitted: May 20, 2015; Accepted: October 14, 2015.

Reference information: J Clin Invest. 2016;126(1):85-98. doi:10.1172/JCI82897. chromosomal translocations, all of these translocations are associated with distinct DNA methylation signatures in primary AML samples, suggesting that alterations in DNA methylation are a common consequence of these chromosomal alterations. In addition, previous studies have suggested a functional relationship between PML-RARA and DNMT3A. PML-RARA is known to act as an oncogenic transcription factor that is capable of initiating leukemia in mouse models (8-14); its ability to cause APL is actually enhanced by overexpression of DNMT3A1 (15). It has been shown to repress target genes by interacting with a corepressor complex that includes the methylated DNA-binding protein MDB1 and DNMT3A (16-23). At the RARB locus, the physical binding of PMLRARA coincides with recruitment of DNMT3A, methylation of the $R A R B$ promoter, and silencing of gene expression (24). However, it is not yet known whether PML-RARA requires DNMT3A to act as an oncogene. Recent ChIP-sequencing (ChIP-seq) studies in AML cell lines have shown that the DNA methylation changes in close proximity to PML-RARA binding sites are relatively subtle $(25,26)$, suggesting that the functional relationship between $P M L$ RARA and DNMT3A on a whole-genome level may be more complex than that observed at the $R A R B$ locus.

To directly test the hypothesis that AML-initiating fusion genes and DNMT3A mutations are mutually exclusive because the fusions may require DNMT3A to exert their activities, we used BM cells from a previously described, constitutive Dnmt $3 a$-null mouse; we tested the ability of three different fusion oncogenes to induce aberrant self-renewal and leukemia in the absence of DNMT3A. Our results show that DNMT3A (but not DNMT3B) is required only for PML-RARA to induce aberrant self-renewal in myeloid progenitor cells and to initiate APL in vivo. DNMT3A is not required for the leukemogenic potential of $M L L-A F 9$ or the self-renewal of myeloid 
A

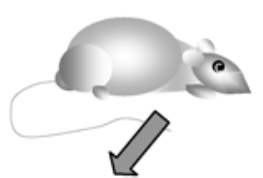

WT

Dnmt3a
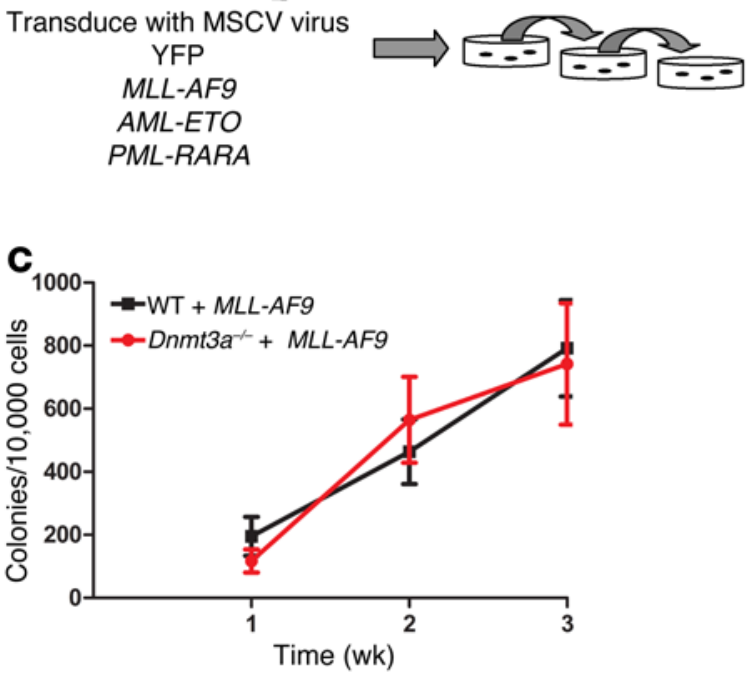

E
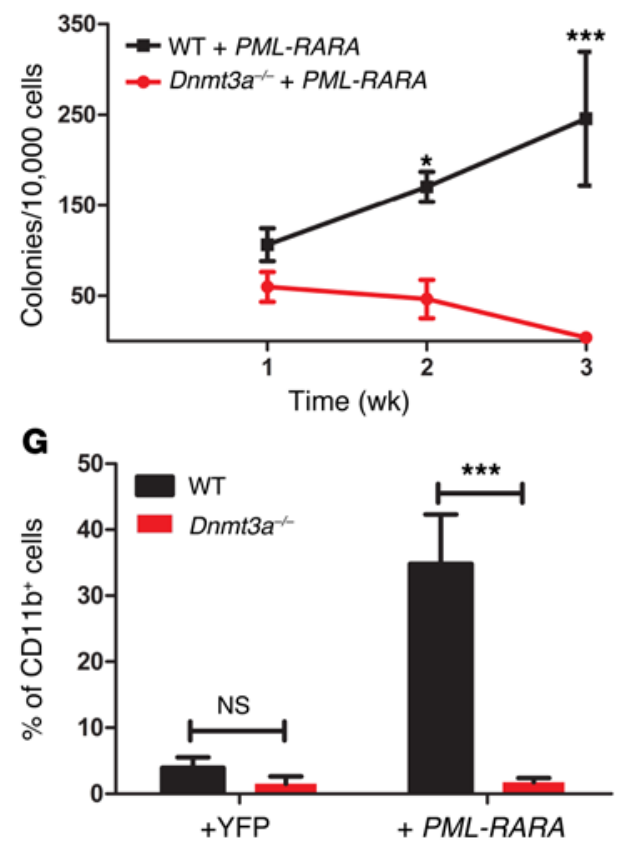

B

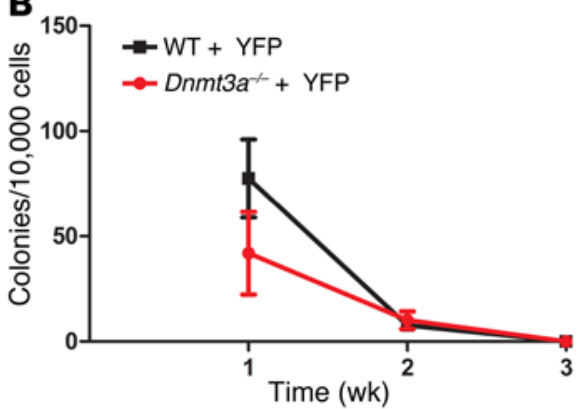

D

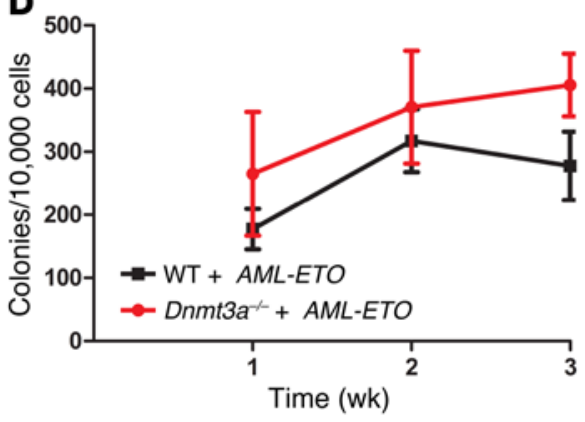

F $\quad W T+P M L-R A R A \quad D n m t 3 a^{-\alpha}+P M L-R A R A$

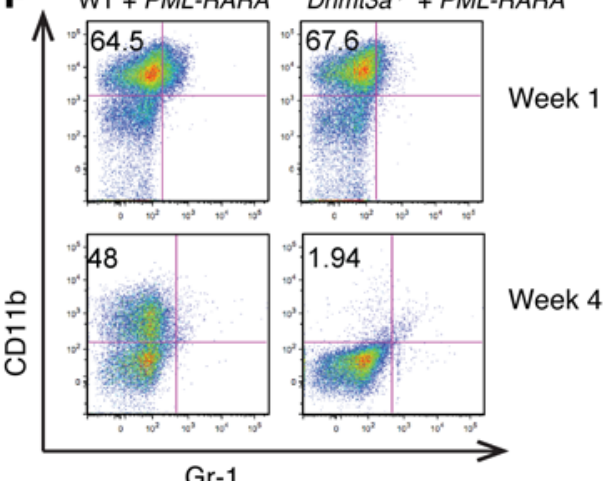

Figure 1. DNMT3A is

required for aberrant self-renewal ability conferred by PML-RARA on hematopoietic progenitor cells. (A) Schematic of the experimental design for results shown in B-E. BM from 2- to 2.5-week-old mice of the indicated genotypes was transduced with the indicated retroviruses, plated in MethoCult media containing IL-3, IL-6, and SCF, and then replated each week. (B) A YFP-expressing control vector did not induce replating in WT or Dnmt3a/- cells. (C and D) Dnmt3a was unnecessary for aberrant self-renewal driven by retroviruses expressing MLL-AFg (C) or RUNX1-RUNX1T1 (AML-ETO) (D). (E) Loss of Dnmt3a eliminated replating driven by a PML-RARA-containing retrovirus. (F) Representative flow cytometric plots for the myeloid markers $\mathrm{Gr}-1$ and CD11b for week 1 versus week 4 of replating. (C) Flow data for CD11b positivity at week 4 of replating, demonstrating loss of myeloid cells in $\mathrm{Dnmt3a^{-1 }}$ marrow transduced with PML-RARA. $n=3-6$ for all experiments. ${ }^{*} P<0.05$ and ${ }^{* * *} P<0.001$ by 2 -tailed, unpaired $t$ test. progenitors induced by RUNX1-RUNX1T1. These data point out the complexity in understanding the mutual exclusivity of AML mutations, but demonstrate one mechanism that may help to explain this finding in acute promyelocytic leukemia (APL).

\section{Results}

Constitutive loss of DNMT3A leads to canonical, locus-specific DNA hypomethylation in hematopoietic cells. For the studies of DNMT3A deficiency in this report, we used a constitutive Dnmt3a-KO mouse that contains a deletion of part of the catalytic methyltransferase domain of the DNMT3A enzyme (27). We verified that this allele was a true null for DNMT3A protein (Supplemental Figure $1 \mathrm{~A}$; supplemental material available online with this arti- cle; doi:10.1172/JCI82897DS1) and that Dnmt3a RNA expression was dramatically reduced, with no effects on neighboring genes (data not shown and see below). Since constitutive Dnmt3a-/mice die at about 3 weeks of age with severe runting (27), we harvested BM cells from WT and Dnmt $3 a^{-/}$mice at 2.5 weeks of age and transplanted them into lethally irradiated C57Bl/6 recipients to study the effects of DNMT3A loss on hematopoiesis. The BM cells were allowed to engraft in these mice for 8 weeks and were then harvested for morphologic examination and flow cytometric and DNA methylation studies. Importantly, there were no detectable perturbations in the populations of the stem/ progenitor cells or mature cells of any lineage in the engrafted BM from the Dnmt $3 a^{-1 /}$ donors (see below). BM cells from 3 WT 

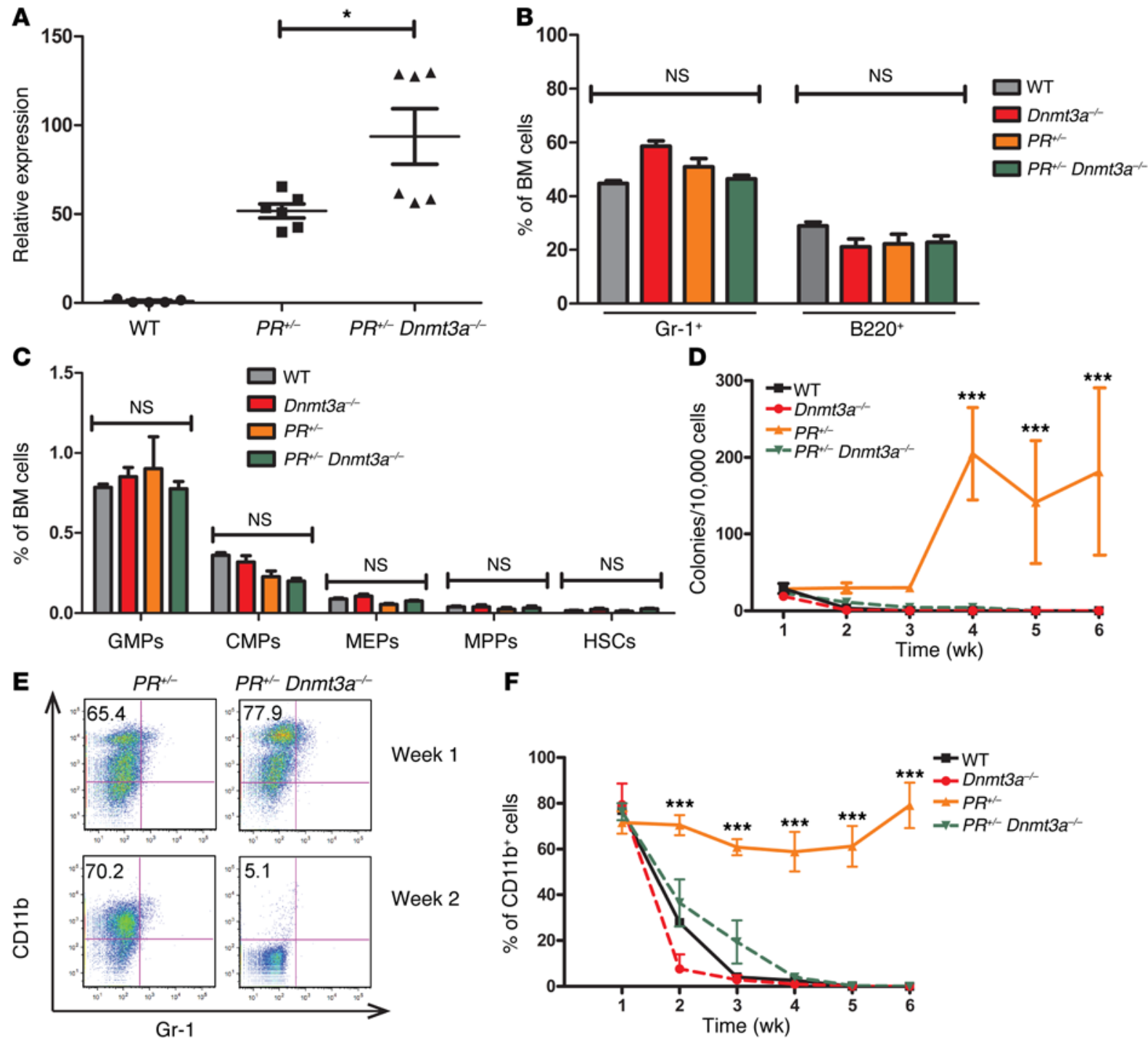

$\mathbf{F}$
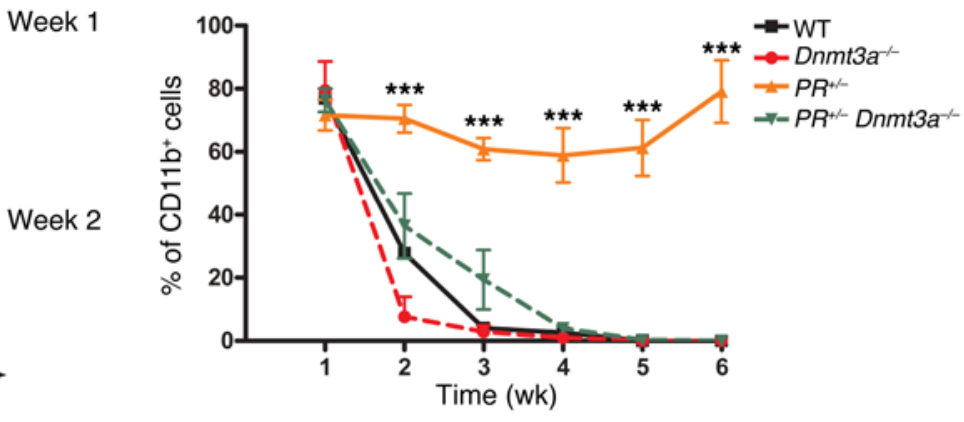

Figure 2. DNMT3A is required for the aberrant self-renewal ability of PML-RARA-expressing mouse BM cells ex vivo. (A) RT-PCR for $P M L-R A R A$ expression in BM cells derived from Ctsg-PML-RARA mice $\left(P R^{+-}\right)$or Ctsg-PML-RARA mice that were also deficient for DNMT3A $\left(P R^{+-} D n m t 3 a^{-/}\right)$. (B and $\left.\mathbf{C}\right)$ BM from 2- to 2.5-week-old mice of the indicated genotypes was transplanted into lethally irradiated WT recipients in a noncompetitive transplantation. (B) Quantification of cell numbers in the mature myeloid compartment (Gr-1+, left panel) versus the mature B cell compartment (B220+, right panel) at

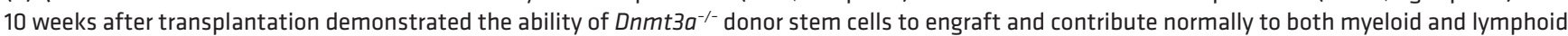
lineages (see also Supplemental Figure 3A). (C) Quantification of the indicated progenitor and stem cell compartments showed no significant differences for any genotype 10 weeks after transplantation. MEPs, megakaryocyte-erythroid progenitors. (D-F) BM from 2- to 2.5-week-old mice of the indicated genotypes was plated in MethoCult media containing IL-3, IL-6, and SCF and replated weekly. (D) Quantification of colony numbers demonstrated a loss of colony formation by $P R^{+/-} D n m t 3 a^{-/-}$cells. (E) Representative flow cytometric plot for the myeloid markers Gr-1 and CD11b demonstrated a loss of myeloid cells from $P R^{+/-} D n m t 3 a^{-1-}$ mice after 2 weeks of replating in MethoCult media. (F) Graph of CD11b positivity over time. $n=3-6$ for all experiments. ${ }^{*} P<0.05$ and ${ }^{* *} P<0.001$ for $P R^{+/-}$versus all other genotypes, by 2 -way ANOVA.

and 3 Dnmt $3 a^{-/}$mice were subjected to CpG capture and bisulfite sequencing. The targeted genomic regions included all annotated CpG islands as well as areas of the genome that have been previously established to show differential patterns of methylation in different tissues and other regulatory DNA sequences such as enhancers and insulators (Supplemental Table 1). The coverage data for each sample are shown in Supplemental Table 2.

All three Dnmt3a-/- BM samples displayed a global decrease in highly methylated CpGs and a corresponding increase in CpGs that were unmethylated (Supplemental Figure 1B). Nearly all of the CpGs that were differentially methylated were hypomethy- lated in the Dnmt3a $a^{-/}$samples (231,001 hypomethylated CpGs versus 5,488 that were more methylated in Dnmt3a-- samples, Supplemental Figure 1C). This hypomethylation phenotype is in general agreement with previously published studies using a conditional Dnmt3a-KO mouse, in which the CpGs from purified stem cell and B cell populations of serially transplanted mice were predominantly hypomethylated $(3,28)$.

Nearly all of the 5,000 most differentially methylated CpGs were hypomethylated in all three Dnmt $3 a^{-1-}$ samples (Supplemental Figure 1D), and most of the canonically hypomethylated CpGs occurred in defined genomic regions $(3,28)$. For example, a region 
A

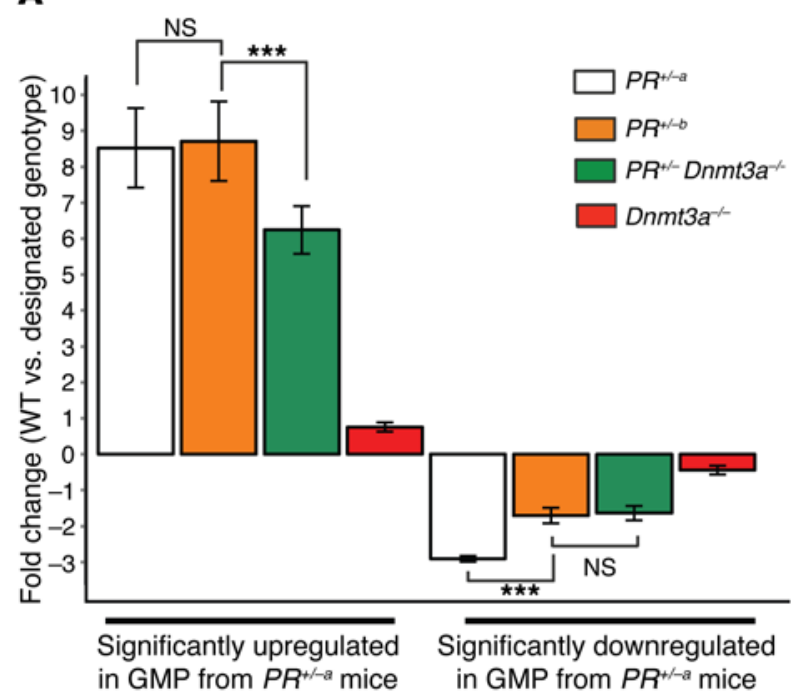

B

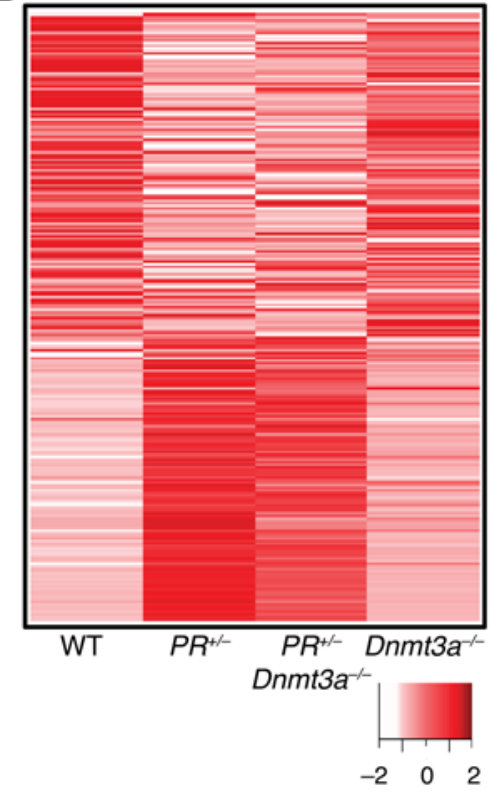

Figure 3. Expression analysis of previously identified PML-RARA-dysregulated genes in GMP cells derived from $\mathbf{P R}^{+/-}, \boldsymbol{P R}^{+/-}$Dnmt3a ${ }^{-/-}$, and $\mathbf{D n m t 3 a ^ { - / - }}$ mice. (A and B) Using Affymetrix Mouse Exon 1.0ST arrays, we interrogated gene expression in GMP cells purified from BM cells of mice transplanted with WT $(n=4), P R^{+/-}$ $(n=2), \mathrm{PR}^{+/-} \mathrm{Dnmt3a}^{-/-}(n=4)$, or Dnmt3a-/- $(n=3)$ marrow cells that were harvested 6-8 weeks later. (A) Mean fold changes for 239 probe sets previously found to be significantly dysregulated in GMP cells derived from the BM of 6- to 8-week-old Ctsg-PML-RARA mice (labeled as $P R^{+-a}$ ) versus WT GMP cells ( $n=4$ for each; ref. 31 and Supplemental Table 3). The mean fold changes (compared with those for WT GMP cells) for the 112 probe sets that were upregulated in the $P R^{+/-a}$ mice are shown in the left panel, and the mean fold changes for the 127 probe sets that were downregulated are shown on the right. Fold changes for the $P R^{+/-b}, P R^{+/-} D n m t 3 a^{-/-}$, and $D n m t 3 a^{-/-}$probe sets were calculated by comparison with the WT data from the current set of experimental data. (B) Heatmap of Z-scored data using the same $239 P R^{+/-}$-dysregulated probe sets defined in Wartman et al. (31) and displaying the average values obtained from the arrays generated from GMP-purified cells with the designated genotype used in this study. The list of genes with significant variation in expression levels was generated using a fold change of 2 and an FDR criterion of 0.05 or less. Bar plot $P$ values ( ${ }^{* *} P<0.001$ ) are based on Student's paired $t$ test, with a 2-tailed distribution.

on chromosome 16 near the internal (P2) promoter of the Runx1 gene contained a "canyon" that was completely unmethylated in both WT and Dnmt $3 a^{-/}$BM cells (Supplemental Figure 1E and ref. 28). This canyon was flanked on both sides by regions that were highly methylated in WT BM cells, but essentially unmethylated in Dnmt3a $a^{-1}$ BM cells.

These results establish that this DNMT3A-deficient mouse strain has a strong focal hypomethylation phenotype in hematopoietic cells, credentialing it for these studies. A complete description of the methylation and expression phenotypes of the BM cells of these mice will be presented elsewhere (Ketkar et al., unpublished observations).

DNMT3A is required for aberrant self-renewal induced by PML-RARA but is dispensable for self-renewal caused by RUNX1RUNX1T1 and MLL-AF9. We next asked whether DNMT3A deficiency influenced aberrant self-renewal induced by the AML fusion genes RUNX1-RUNX1T1, MLL-AF9, and PML-RARA. These oncogenes are capable of inducing an aberrant selfrenewal phenotype ex vivo when expressed in WT mouse BM cells with retroviral vectors $(13,29,30)$. Whole BM cells, when transduced with mouse stem cell viruses (MSCVs) containing cDNAs for RUNX1-RUNX1T1, MLL-AF9, or PML-RARA and then plated in semi-solid MethoCult media, form CFU granulocyte-monocyte (CFU-GM) colonies that express CD11b (a marker of terminally differentiated myelomonocytic cells). Progenitor cells from these transductions can be serially replated for several weeks. WT BM cells do not serially replate in this assay, thereby losing the ability to form new myeloid colonies containing cells that express CD11b after 1 week. To test whether self-renewal in this assay was dependent on Dnmt3a, we harvested whole BM cells from 2to 2.5-week-old WT or Dnmt3a-/littermates and transduced the cells with MSCVs containing either an empty vector with an internal ribosome entry site-yellow fluorescent protein (IRESYFP) cassette or with viruses containing cDNAs for RUNX1RUNX1T1, MLL-AF9, or PMLRARA (and an IRES-YFP cassette to identify the transduced cell populations). Transduced cells were plated in MethoCult media (Figure 1A). Each week, colonies were quantified, and cells were assessed for expression of $\mathrm{CD} 11 \mathrm{~b}$ by flow cytometry, and then cells were replated. Both WT and Dnmt3a $a^{--}$BM cells transduced with empty control YFP vector lost the ability to form colonies after one or two replating cycles (Figure 1B). In contrast, both WT and Dnmt $3 a^{-1-}$ BM transduced with either MLL-AF9 or RUNX1RUNX1T1 gave rise to increasing numbers of colonies over time (Figure 1, C and D). In contrast, the PML-RARA-expressing virus could induce serial replating in WT- but not Dnmt3a/-derived BM cells (Figure 1E). Both WT and Dnmt $3 a^{-1-} \mathrm{BM}$ transduced with either the MLL-AF9 or RUNX1-RUNX1T1 viruses maintained expression of the myeloid marker CD11b after three replatings; in contrast, CD11b expression was maintained in WT BM transduced with PML-RARA, but was lost in the absence of Dnmt3a (Figure 1, F and G). Additional flow cytometric analysis and morphologic examination demonstrated that the few remaining cells at week 3 in the Dnmt $3 a^{-1-}$ BM transduced with PML-RARA were $\mathrm{Fc}_{\mathrm{N}} \mathrm{R}^{+}, \mathrm{c}-\mathrm{Kit}^{\mathrm{hi}}$ mast cells, similar to cells transduced with the empty vector GFP (data not shown). Together, these results indicate that in a retroviral transduction system, aberrant myeloid self-renewal by PML-RARA requires $D n m t 3 a$, whereas self-renewal driven by $M L L-A F 9$ or RUNX1-RUNX1T1 does not.

$D N M T 3 A$ is required for the aberrant self-renewal of hematopoietic progenitor cells from Ctsg-PML-RARA mice. To orthogonally validate these results, we crossed $D n m t 3 a^{+/}$mice with a well-charac- 
A

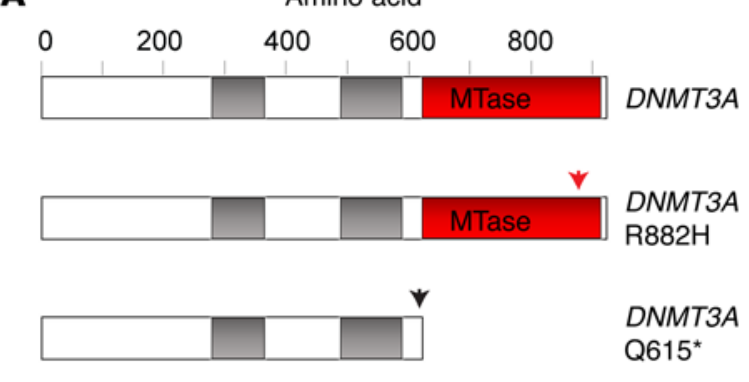

B

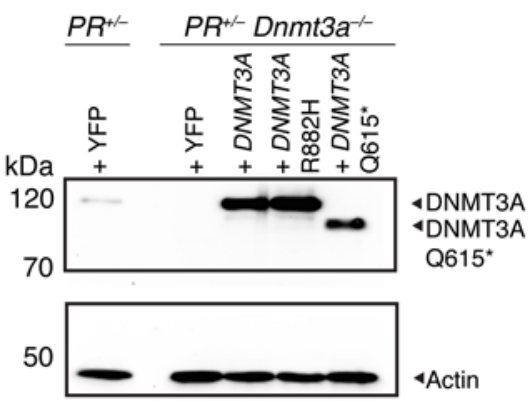

C
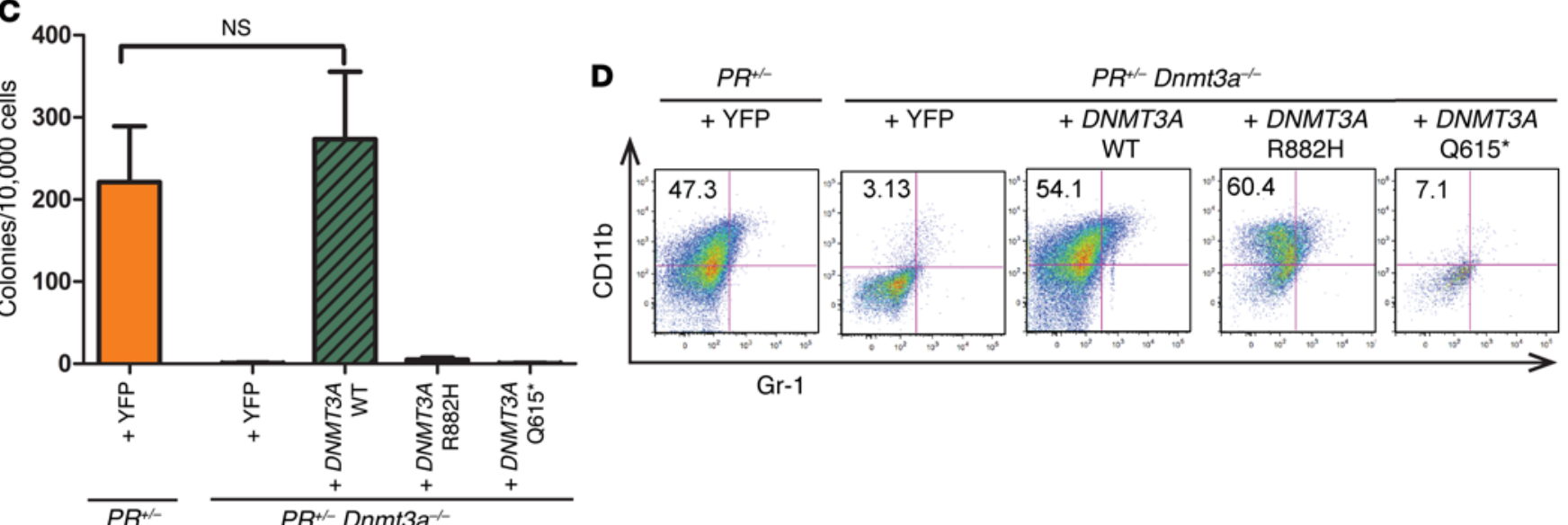

E

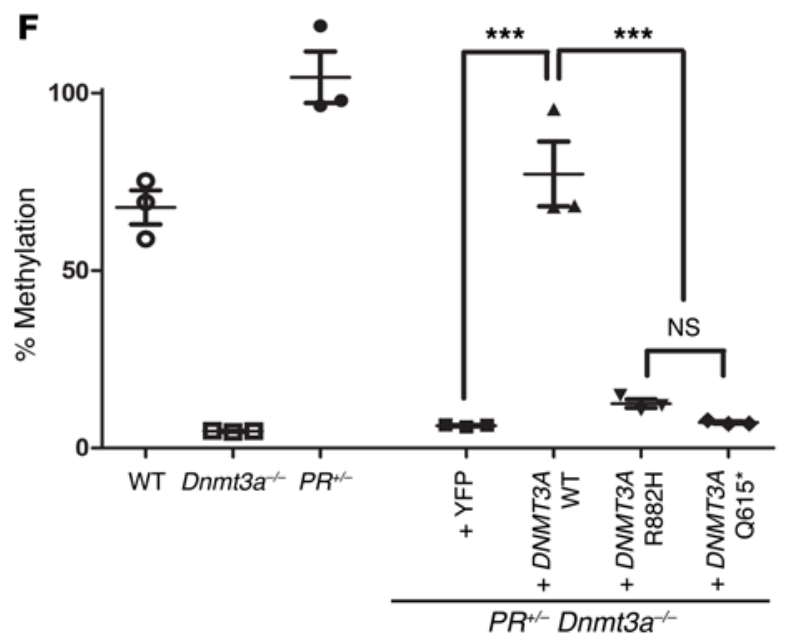

Figure 4. DNA methyltransferase activity of DNMT3A is required for aberrant self-renewal by PML-RARA ex vivo. (A) Schematic of human DNMT3A cDNAs used in B-E. Mutations in the methyltransferase domain (MTase) from AML patients induced loss of catalytic function (R882H, red arrow) or lacked the methyltransferase domain altogether (Q615*, black arrow). (B) Western blot from $P R^{+/-}$or $P R^{+/-} D n m t 3 a^{-/-}$BM cells transduced with retroviruses containing human DNMT3A cDNAs and IRES-YFP and then sorted for YFP+ cells. Cells were harvested after 1 week in MethoCult medium. The positions of full-length and truncated DNMT3A are indicated on the right. (C) BM from 2- to 3-week-old mice was transduced with the indicated viruses, and GFP+ cells were sorted and serially replated in MethoCult medium. Week-4 colony numbers demonstrated that only WT DNMT3A with intact DNA methyltransferase activity was able to restore the colony-forming ability to $P R^{+/-} D n m t 3 a^{-/-}$myeloid cells. (D) Flow cytometric analysis of week-4 MethoCult cells demonstrated a persistence of CD11b expression in $P R^{+/-} D n m t 3 a^{-/-}$cells, conferred only by WT DNMT3A. (E) Graph of CD11b expression percentages at 4 weeks of serial replating in MethoCult medium. (F) Assay of methylation at a specific Hpall site in the Runx 1 locus that is dependent on DNMT3A for methylation in WT BM cells (Site 1 in Supplemental Figure 1E). Reexpression of WT, full-length DNMT3A by retroviral transduction restored a normal level of methylation to these sites after 7 days in MethoCult media. Retroviruses containing mutant DNMT3A (R882H or Q615*) did not restore methylation at this site. $n=3-4$ for all experiments. ${ }^{*} P<0.05,{ }^{* *} P<0.005$, and ${ }^{* * *} P<0.001$, by 2-way ANOVA.

terized $P M L-R A R A$-expressing transgenic mouse model created in our laboratory (Ctsg-PML-RARA, hereafter referred to as $P R^{+/-}$) (12) to ultimately generate $P M L-R A R A$-expressing mice lacking both copies of Dnmt3a $\left(P^{+/-} \mathrm{Dnmt} 3 \mathrm{a}^{-/-}\right)$as well as all relevant control genotypes (WT, Dnmt3a/-, and $P R^{+/}$).
Ctsg-PML-RARA mice express a human $P M L-R A R A$ fusion gene under control of the endogenous mouse cathepsin $G$ locus, which leads to $P M L-R A R A$ expression in early myeloid progenitor cells (with highest expression levels in granulocyte-macrophage progenitors [GMPs], in which dysregulation of target genes is most pro- 
A

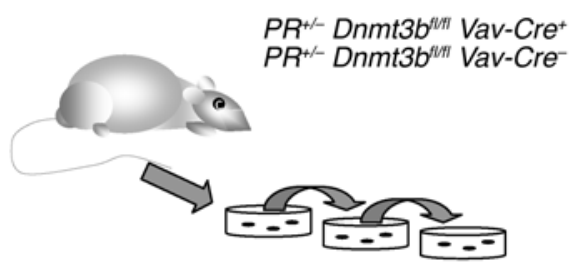

C $P R^{+/-D n m t 3 b^{n / m}} P R^{+-}$Dnmt3b

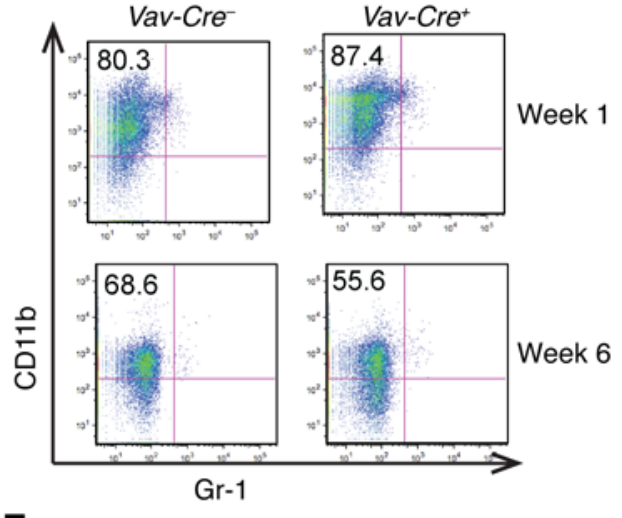

$\mathbf{E}$

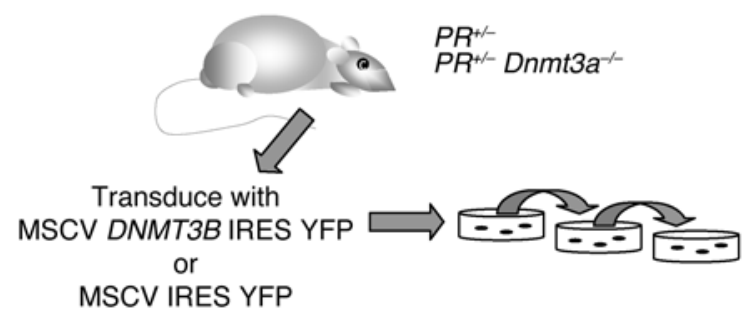

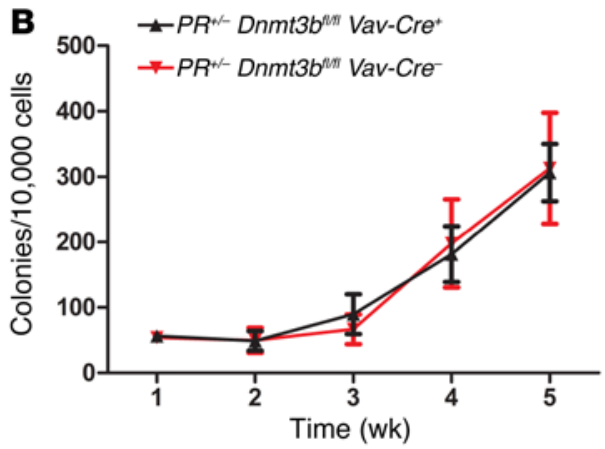

D
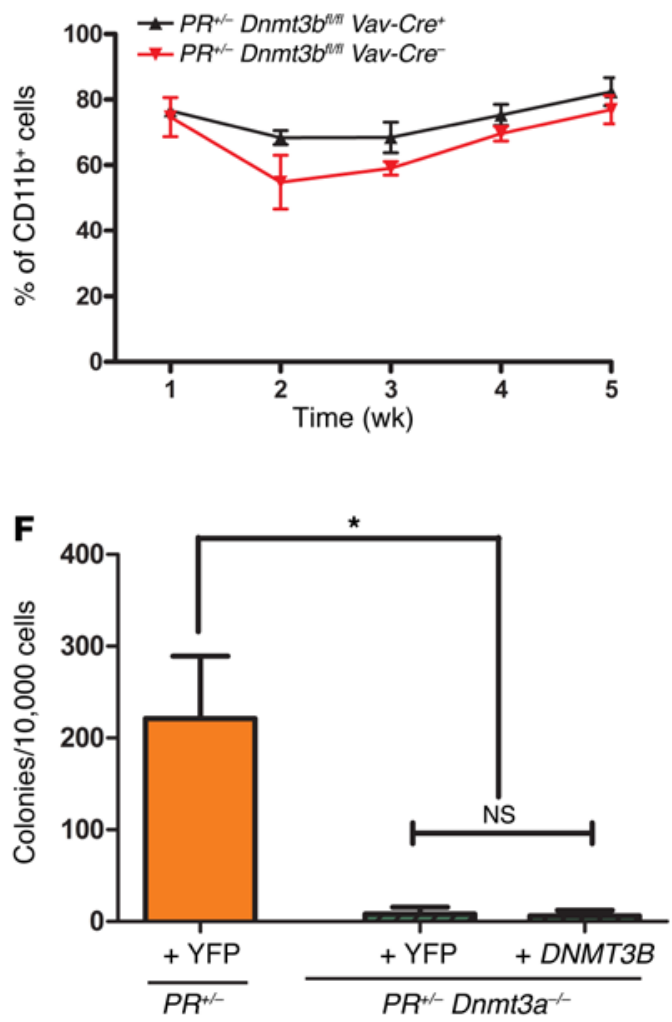

Figure 5. DNMT3B is not required for the aberrant self-renewal ability conferred by PML-RARA in murine BM progenitor cells. (A) Schematic of the experimental design for the results shown in B-D. BM from 2- to 2.5-week-old mice of the indicated genotypes was plated in MethoCult media containing IL-3, IL-6, and SCF and replated each week. (B) Quantification of colony numbers demonstrated no difference in colony formation in $P R^{+/-} D n m t 3 b^{f / / f l}$ cells with or without Vav-Cre. (C) Representative flow cytometric plot for the myeloid markers Gr-1 and CD11b after 6 weeks of MethoCult replating demonstrated that self-renewal of myeloid cells from $P R^{+/-}$mice was not dependent on Dnmt3b. (D) Graph of CD11b positivity over time. (E) Schematic of the experimental design for the results shown in F. Marrow from 2- to 2.5-week-old mice of the indicated genotypes was retrovirally transduced with MSCV vectors containing WT DNMT3B-IRES-YFP or YFP only and then sorted for YFP+ ${ }^{+}$cells and plated in MethoCult media as in $\mathbf{A}$. (F) Quantification of colony numbers at week 4 illustrates that overexpression of $D N M T 3 B$ was not able to rescue aberrant self-renewal in $P R^{+/-} D n m t 3 a^{-/-} B M$ cells. $n=3-6$ for all experiments. ${ }^{*} P<0.05$, by 2 -tailed, unpaired $t$ test.

nounced) (31) and the development of lethal APL with long latency and high penetrance $(\sim 60 \%$ at 1 year in $\mathrm{C} 57 \mathrm{Bl} / 6$ mice $)(12)$. BM cells derived from these mice display an advantage with competitive transplantation and give rise to self-renewing myeloid progenitors on semi-solid medium ex vivo (31). We reasoned that if functional Dnmt $3 a$ were required for any of these aberrant self-renewal phenotypes, they would be abrogated in $P R^{+/-} D n m t 3 a^{-/}$mice.

We first measured PML-RARA expression by quantitative reverse transcriptase PCR (qRT-PCR) in BM cells derived from $\mathrm{WT}, \mathrm{PR}^{+/}$, and $\mathrm{PR}^{+/-}$Dnmt3a $3 \mathrm{a}^{-/}$mice (Figure 2A) to determine whether DNMT3A deficiency altered the expression of $P M L$ -
RARA. We detected no PML-RARA transcripts in WT BM cells, confirming the specificity of this assay (31). PML-RARA expression levels were not reduced by DNMT3A deficiency and were in fact slightly higher than those detected in $P R^{+-}$mice.

We next assessed the engraftment and differentiation potential of Dnmt3a-/- versus $\mathrm{PR}^{+-}$Dnmt3a/- donor cells (compared with WT and $P R^{+/-}$donor cells) by harvesting whole BM from 2- to 2.5-week-old littermates from all four genotypes and transplanting these cells into lethally irradiated WT recipients. After 8 to 10 weeks, the engrafted BM cells from all donor genotypes were assessed for their contributions to myeloid and lymphoid lineages 
A
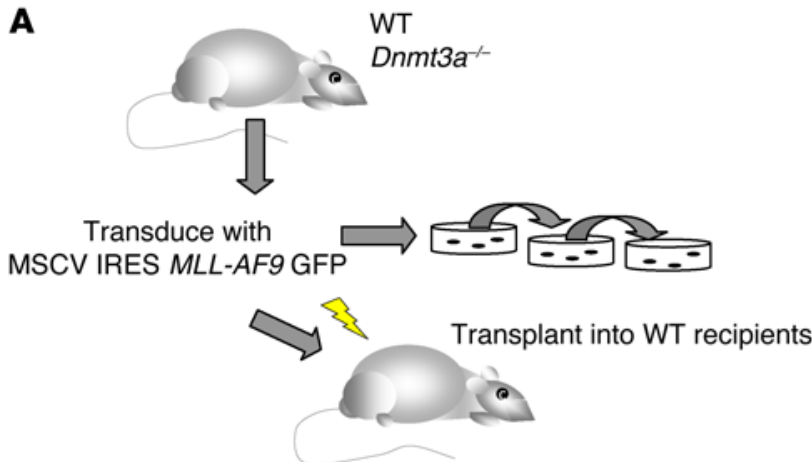

C

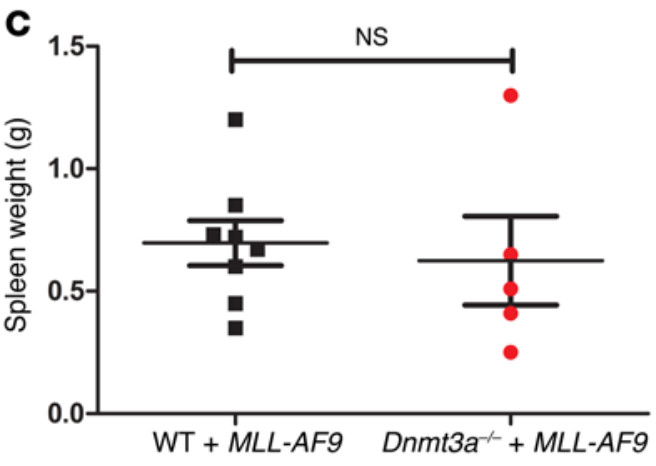

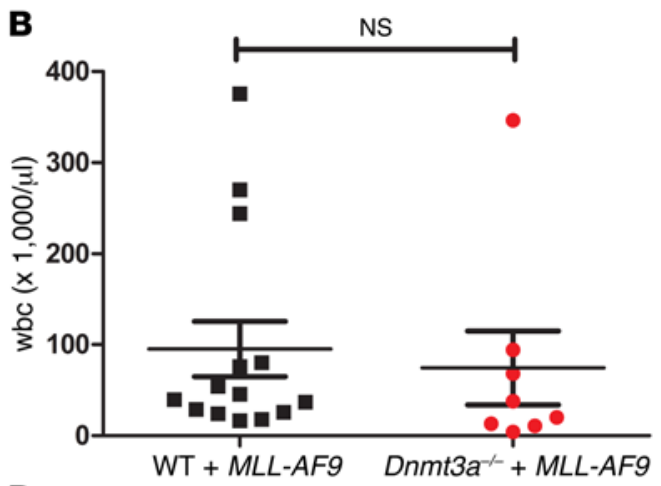

D

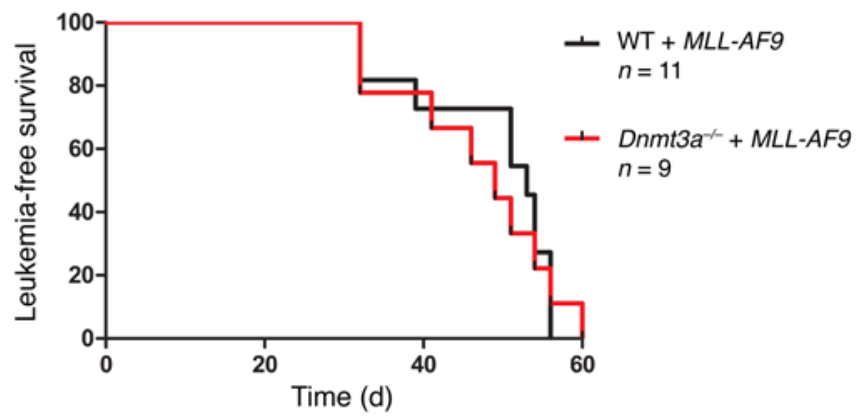

Figure 6. DNMT3A is dispensable for leukemia induced by MLL-AF9 overexpression. (A) Schematic of the experimental design for the results shown in B-E. BM from 2- to 3-week-old mice of the indicated genotypes was harvested and transduced with an MLL-AF9-expressing retrovirus before transplantation into lethally irradiated WT mice. (B) wbc counts 28 days after transplantation demonstrated an equal degree of leukocytosis in recipients of MLL-AF9transduced WT and Dnmt3a- BM. (C) Spleen weights of moribund animals were not significantly different, regardless of DNMT3A status. (D) MLL-AFg was able to initiate lethal leukemia with $100 \%$ penetrance and equal latency using BM cells with or without Dnmt3a ( $n=11$ for WT + MLL-AF9; $n=9$ for Dnmt3a $a^{-1-}+$ MLL-AF9). NS, by 2-tailed, unpaired $t$ test.

(Figure 2B). We observed no significant difference in the proportion of mature myeloid $\left(\mathrm{Gr}-1^{+}\right.$and/or CD11b $)$or mature lymphoid $\left(\mathrm{B}^{2} 2 \mathrm{O}^{+}\right.$or $\left.\mathrm{CD}^{+}\right)$cells, regardless of Dnmt3a status. Similarly, there were no differences in the numbers of stem and progenitor cells in recipients of marrow with or without DNMT3A (Figure 2C). Sixteen weeks after transplantation, we observed no differences in the numbers of PB leukocytes, rbc, or platelets, regardless of the presence or absence of DNMT3A (Supplemental Figure $2 \mathrm{~A})$. Histologic examination of the BM cells of engrafted recipients demonstrated normal cellular morphology, with no evidence of dysplastic changes (data not shown). Collectively, these data demonstrate that Dnmt3a loss does not lead to impaired engraftment or grossly altered steady-state hematopoiesis for at least 4 months after transplantation into WT recipient mice.

We next tested whether ex vivo self-renewal was directly altered in BM cells derived from 2- to 2.5-week-old mice of all genotypes (i.e., not transplanted, Figure 2D). We found that WT cells lost the ability to form colonies after one or two replatings, whereas $P R^{+-}$marrow gave rise to increasing numbers of colonies with serial replating. $P R^{+/-} D n m t 3 a^{-/}$cells gave rise to colony numbers similar to those in WT and Dnmt3a $a^{--}$cells with the first and second platings, and likewise failed to replate thereafter. $P R^{+/}$cells maintained CD11b expression with serial replating (Figure 2E). In

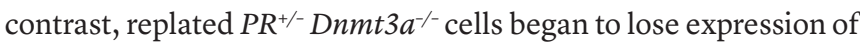
CD11b by the end of the second week (Figure 2, E and F). We also tested whether this phenotype was intrinsic to the hematopoietic compartment (as opposed to an effect from Dnmt3a-/ stromal cells) by transplanting BM from young (2- to 2.5-week-old) mice into lethally irradiated WT C57Bl/6 recipients and harvesting marrow for replating assays 10 weeks after transplantation (Supplemental Figure 2B). As expected, BM derived from the recipients of $P R^{+/}$donors formed colonies with serial replating, whereas BM derived from $P R^{+/}$Dnmt3a $a^{--}$donors did not (Supplemental Figure 2C). These data demonstrate that this phenotype is due to a cell-autonomous defect in the hematopoietic compartment.

DNMT3A deficiency attenuates the expression of genes that are normally upregulated by PML-RARA in GMPs. To determine whether DNMT3A loss affects genes that are specifically dysregulated by PML-RARA, we analyzed the expression of genes previously shown to be differentially expressed between WT and Ctsg-PML$R A R A\left(P R^{+/}\right)$mice (31). In that study, we observed that these genes show the greatest differences in the GMP compartment of $P R^{+/}$mice, where Ctsg is maximally expressed: 112 probesets from the Affymetrix mouse exon ST1.0 arrays were significantly upregulated by PML-RARA in an ANOVA analysis (fold change $\geq 2.0, \mathrm{FDR}<0.05$ ), and 127 probesets were downregulated (31). We therefore performed expression profiling using purified GMP cells from the engrafted marrow of several donor mice of all four genotypes (WT, $n=4 ; P R^{+/}, n=2 ; P R^{+/} D n m t 3 a^{-/}, n=4$; and Dnmt $3 a^{-/}$, $n=3)$ harvested 6-8 weeks after transplantation. The results of these analyses are highly concordant with the results from Wartman et al. (31). Of the 112 upregulated probe sets (designated as the 
A $P R^{+1}$ Dnmt3a- WT competitor $P R^{+/}$Dnmt3a- $\quad($ Ly5.1)
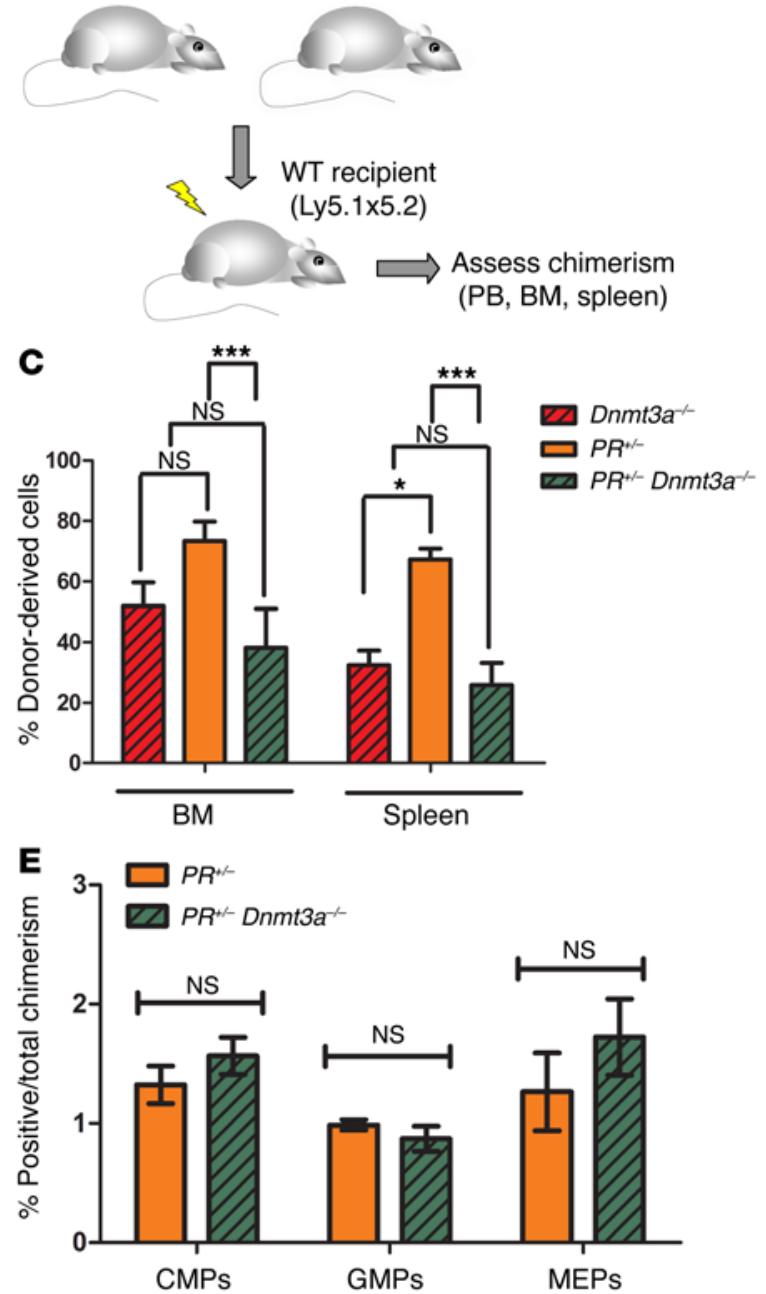
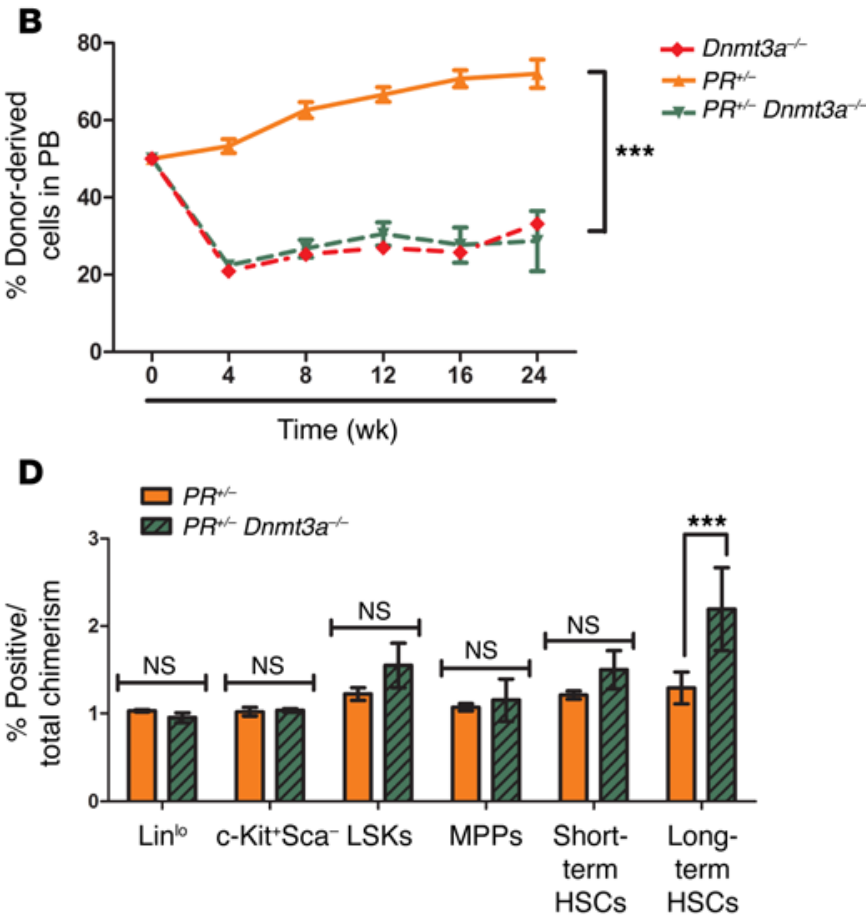

$\mathbf{F}$

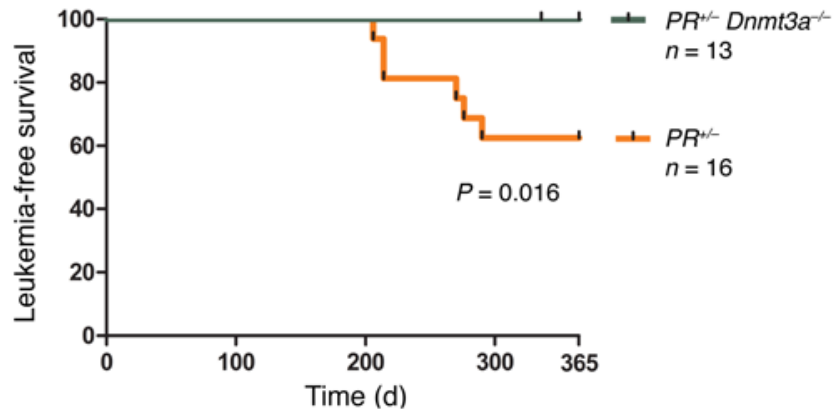

Figure 7. DNMT3A is required for the competitive repopulation advantage conferred by PML-RARA and its ability to induce APL in vivo. (A) Schematic of the experimental design for the results shown in B-E, in which marrow from 2- to 2.5-week-old mice of the indicated genotypes was mixed with WT competitor marrow and transplanted into lethally irradiated recipients (week 0 ) for monitoring of the relative contribution to PB cells, BM, and spleen ( $n=4-21$ per genotype). (B) Flow cytometry at the indicated weeks after transplantation demonstrated that the competitive advantage for $P R^{+/-} B M$ in

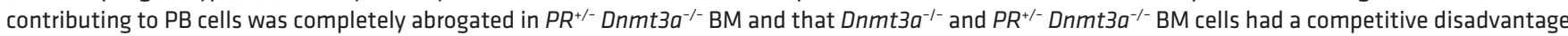
compared with that of WT BM cells in this assay. (C) Examination of chimerism in BM and spleen at 6 months after transplantation indicated a decreased contribution of $P R^{+/-} \mathrm{Dnmt3a^{-/- }}$ marrow compared with that observed for $P R^{+/-}$marrow in both compartments. (D) Characterization of the stem/progenitor compartments in chimeric mice 10 weeks after competitive transplantation. The composition of all compartments was the same for both genotypes, except that $P R^{+/-} D n m t 3 a^{-/-}$donors displayed a significantly increased contribution to the long-term hematopoietic stem cell compartment. LSKs, $\mathrm{Lin}^{10} \mathrm{Sca}{ }^{+} \mathrm{C}-\mathrm{Kit}{ }^{+}$cells. (E) Quantification of myeloid progenitor compartments demonstrated no significant differentiation biases in either genotype. (F) Long-term tumor watch of WT animals transplanted with $P R^{+/-}$or $P R^{+/-} D n m t 3 a^{-/-} \mathrm{BM}$ demonstrated that 6 of the 16 recipients of $P R^{+/-}$and 0 of the 13 recipients of $P R^{+/-} D n m t 3 a^{-/-} \mathrm{BM}$ had succumbed to APL by 1 year after transplantation $(P=0.0336$ by Mantel-Cox test). NS indicates that no differences between any 2 genotypes were statistically significant by 2 -way ANOVA (B) or 1-way ANOVA (C-E). ${ }^{*} P<0.05$ and ${ }^{* * *} P<0.001$, by 1 - or 2 -way ANOVA.

$P R^{+/-a}$ data set in Figure 3), 96 (86\%) exhibited a similar 2-fold or greater increase in expression in the data obtained for this study (denoted as the $P R^{+/-b}$ dataset, Figure 3A and Supplemental Table 3). The average fold change (comparing WT with $P R^{+-}$) for this set of probes in the $P R^{+/-a}$ data set was $8.52 \pm 1.10$ (SEM), which was not significantly different from the average fold change of the same genes in the $P R^{+/-b}$ data set $(8.71 \pm 1.11 ; P=0.75$; Figure $3 \mathrm{~A}$, left panel). GMPs from the $P R^{+/-} D n m t 3 a^{-/-}$mice demonstrated significantly less upregulation of many of these probes: the average fold change was $6.24 \pm 0.66\left(P=9.73 \times 10^{-6}\right.$ compared with $P R^{+/-b}$ mice $)$. These data suggest that DNMT3A loss attenuates the expression of a set of genes that are normally upregulated by PML-RARA in GMP cells. Of the 127 probes that were downregulated by at least 2 -fold in the $P R^{+/-a}$ data set, 107 (84\%) were likewise downregulated in the $P R^{+/-b}$ data set, but to a lesser extent (Figure 3A, right). The average level of downregulation was only -2.91 -fold for the $P R^{+/-a}$ data set, and -1.71 -fold for the $P R^{+/-b}$ data set. The average expression of these downregulated genes was not appreciably attenuated by 
DNMT3A loss, perhaps because the fold changes were relatively small and therefore more difficult to detect. A heatmap of probe set-level data is shown for the 239 dysregulated probes defined by the $P R^{+/-a}$ data set (Figure 3B), plotting only the average values for the data generated in this study for mice with the designated genotypes. These data extend the fold change data shown in Figure $3 \mathrm{~A}$ and demonstrate that a large number of $\mathrm{PR}^{+/-}$-dysregulated probe sets are affected by DNMT3A loss. As expected, GMPs from Dnmt3a $a^{-/-}$mice had very few changes in expression of the genes that are canonically dysregulated by PML-RARA. We also independently defined differentially expressed genes from the four genotypes using only the data from this study (Supplemental Figure 3 and Supplemental Table 4). As expected, these data corroborated the Wartman et al. study (31) for the $P R^{+/-}$-dysregulated genes and identified Dnmt $3 a$ itself as one of the most downregulated genes in the Dnmt $3 a^{-/-}$and $\mathrm{PR}^{+/-}$Dnmt3a $a^{-/-}$GMP cells. The expression signature for Dnmt3a-deficient GMP cells was subtle and limited to very few probe sets, which is consistent with other studies of both mouse and human hematopoietic cells with reduced or absent Dnmt3a expression $(1-3,5)$.

DNA methyltransferase activity of DNMT3A is required for aberrant self-renewal by PML-RARA ex vivo. Both DNMT3A and the highly homologous enzyme DNMT3B are known to have de novo DNA methyltransferase activities (32). The most common DNMT3A mutations in AML occur at residue R882 (2). DNMT3A possessing the $\mathrm{R} 882 \mathrm{H}$ mutation has been shown to exhibit reduced DNA methyltransferase activity and produce a dominant-negative effect on WT DNMT3A, which leads to a focal DNA hypomethylation phenotype in primary AML samples (6). Some other mutations, such as the Q615* mutation, introduce a premature stop codon that is predicted to form a truncated protein lacking the entire C-terminal methyltransferase domain (Figure 4A and ref. 2). However, DNMT3A has also been reported to influence gene expression by interacting with and stabilizing a corepressive complex in a manner that is independent of its methyltransferase activity (33). To determine whether the DNA methyltransferase activity of DNMT3A is necessary for PML-RARA functions, we conducted complementation experiments in which $\mathrm{PR}^{+-} \mathrm{Dnmt} 3 \mathrm{a}^{-/}$cells were transduced with retroviruses containing either full-length WT human DNMT3A, full-length DNMT3A with the R882H mutation, the Q615* truncation mutant, or an empty IRES-YFP vector control. We verified that all constructs were expressed in $\mathrm{PR}^{+/-} \mathrm{Dnmt3a/-} \mathrm{BM}$ cells that were sorted for transduced $\left(\mathrm{YFP}^{+}\right)$ cells using Western blotting with an $\mathrm{Ab}$ against the $\mathrm{N}$-terminus of DNMT3A (Figure 4B). All proteins were highly expressed and migrated at their predicted sizes on SDS-PAGE gels. When sorted $\mathrm{YFP}^{+}$cells were plated in MethoCult medium, $\mathrm{PR}^{+/}$Dnmt $3 a^{-/}$cells transduced with the empty YFP vector ceased to produce colonies by week 4. Reintroduction of WT DNMT3A was able to restore both colony formation (Figure 4C) and CD11b expression (Figure 4, D and E). However, neither of the two mutants known to be deficient for DNA methyltransferase activity (DNMT3A R882H and $D N M T 3 A$ Q615*) was able to restore the replating ability (Figure $4 \mathrm{C}$ ) or expression of CD11b (Figure 4, D and E).

We also asked whether WT DNMT3A was able to restore the normal methylation of a subset of CpG residues in the Runx1 P2 promoter region shown in Supplemental Figure 1E (green num- bers 1-4 shown above the gene). We developed a set of PCR amplicons that each contained a single site for the methylation-sensitive restriction endonuclease HpaII (i.e., a single assayable $\mathrm{CpG}$ residue, see Supplemental Table 5). We obtained DNA from BM

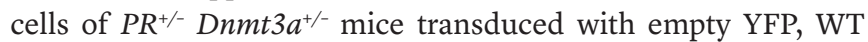
DNMT3A, or the catalytically impaired DNMT3A mutants and cultured the transduced $\mathrm{YFP}^{+}$cells in MethoCult media for 6 or 7 days. DNA harvested from these cells (and from the appropriate control mice) was then digested to completion with HpaII, and qPCR was performed to assess the total level of HpaII cleavage within each amplicon (as a surrogate for the level of cytosine methylation at that particular CCGG cleavage site). Compared with WT or $P R^{+/-}$-derived cells, $P R^{+/-}$Dnmt $3 a^{-/-}$cells transduced with empty YFP displayed near-complete hypomethylation at site 1 (shown in Figure 4F) and site 2 (Supplemental Figure 4B) within the Runx1 P2 promoter region. Reintroduction of WT DNMT3A, but not the $\mathrm{R} 882 \mathrm{H}$ or $\mathrm{Q} 615^{*}$ mutants, restored a near-normal level of methylation to these two sites after only 1 week, a time frame corresponding to the rapid reintroduction of self-renewal by expression of WT DNMT3A. Sites 3 and 4, in the hypomethylation "canyon" that is normally unmethylated in BM cells, did not display remethylation with overexpression of DNMT3A for 1 week (Supplemental Figure 4, A-C); these controls demonstrate that HpaII was capable of cleaving unmethylated sites in these DNA samples and that the overexpression of DNMT3A did not cause abnormal methylation of CpGs that are not normally methylated. Together, these results strongly suggest that it is the DNA methyltransferase activity of DNMT3A, per se, that is required for PML-RARA to drive aberrant self-renewal in myeloid progenitor cells.

$D N M T 3 B$ is dispensable for the aberrant self-renewal ability of PML-RARA mouse BM ex vivo. DNMT3B exhibits sequence specificity that is different from that of DNMT3A in vitro (34), but ChIPseq studies have demonstrated extensive overlap in the genomic regions bound by DNMT3A and DNMT3B in vivo (35). To determine whether PML-RARA also requires DNMT3B for its ability to induce self-renewal, we generated $P R^{+/-}$mice that were null for the Dnmt $3 b$ allele in BM cells. Because Dnmt3b loss leads to embryonic lethality, we crossed mice with floxed Dnmt3b alleles (36) with mice expressing the pan-hematopoietic Vav1-Cre transgene (37) to generate animals with a selective loss of $D n m t 3 b$ in hematopoietic cells. This conditional null allele of $D n m t 3 b$ has previously been demonstrated to result in no production of DNMT3B protein by Western blot analysis (38). We intercrossed these three strains of mice to generate $P R^{+/-}$Dnmt3 $b^{f / f l}$ mice with or without Vav1-Cre

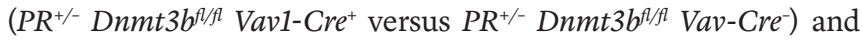
then compared the ability of BM cells from these mice to selfrenew ex vivo, as described above (Figure 5A). Importantly, total BM cells from Dnmt $3 b^{f / f l} \mathrm{Vav1}^{-\mathrm{Cr}^{+}}$mice demonstrated greater than 95\% floxing efficiency of Dnmt3b (data not shown) and had a focal hypomethylation phenotype that was overlapping but distinct from that of DNMT3A-deficient mice (ref. 7 and Ketkar et al., unpublished observations). Dnmt3b was not required for $P M L-$ $R A R A$ to drive self-renewal, since $P R^{+/-} D n m t 3 b^{f / f l} \mathrm{BM}$ could be serially replated and formed equal numbers of colonies regardless of the presence or absence of Cre-mediated Dnmt3b excision (Figure $5 \mathrm{~B}$ ). In addition, Dnmt $3 b$ loss was essentially dispensable for maintaining CD11b expression with serial replating (Figure 5C), 
although $\mathrm{PR}^{+/-} \mathrm{Dnmt} 3 \mathrm{~b}^{f / f l} \mathrm{Vav}$-Cre ${ }^{+}$-positive cells displayed a slight decrease in CD11b positivity that was statistically significant over time $(P<0.05$ by 2 -way ANOVA; Figure $5 D)$. We also performed a complementation experiment by transducing $\mathrm{PR}^{+-}$Dnmt3a-/$\mathrm{BM}$ cells with a retrovirus expressing a full-length WT human DNMT3B cDNA (Figure 5E and Supplemental Figure 5). Overexpression of DNMT3B was unable to restore colony formation (Figure $5 \mathrm{~F}$ ). These results reinforce the hypothesis that the requirement of PML-RARA for DNMT3A is specific for the methyltransferase activity of this protein, and it cannot be replaced by DNMT3B.

DNMT3A is dispensable for leukemia induction by $M L L-$ $A F 9$. The replating results shown above revealed that aberrant self-renewal by $M L L-A F 9$ and RUNX1-RUNX1T1 did not require Dnmt3a. We next tested whether $M L L-A F 9$ leukemia induction was Dnmt $3 a$ independent in vivo. We expressed $M L L-A F 9$ cDNA in WT or Dnmt3a-/- BM cells via retroviral transduction and then transplanted these cells into lethally irradiated $\mathrm{WT}$ recipients (Figure 6A). This retroviral model of $M L L-A F 9$ produces a rapid-onset, high-penetrance AML (30). Four weeks after transplantation, recipients of $M L L-A F 9$-transduced BM showed elevated wbc counts, regardless of the genotype of the transduced donor cells (Figure 6B), and succumbed shortly thereafter to AML, with identical latency, similar degrees of splenomegaly, and $100 \%$ penetrance (Figure 6, C and D). We observed no detectable phenotypic differences between the leukemias that arose in WT versus Dnmt3a/- BM.

$D N M T 3 A$ is required for a $P M L-R A R A$-driven competitive advantage and APL development in vivo. Previous studies using a conditional Dnmt3a-deficient mouse model revealed a progressive deficit in hematopoietic maturation in serial competitive transplants (3). In contrast, $P R^{+/-}$-derived BM cells have a competitive transplantation advantage, as demonstrated by an increased ability to contribute to peripheral blood lineages (especially myeloid) over time (39). To determine whether Dnmt3a is required for this competitive advantage, we mixed whole BM cells from mice with the indicated genotypes (Figure 7A) at a ratio equal to that of WT competitor BM cells and transplanted the cells into lethally irradiated WT mice. Serial analyses of $\mathrm{PB}$ at 4 -week intervals confirmed that $P R^{+/-} \mathrm{BM}$ was able to outcompete WT marrow over time, with $72 \%$ $\pm 3.7 \%$ (SEM) of all $\mathrm{PB}$ cells being derived from $\mathrm{PR}^{+/}$donor marrow at 16 weeks after transplantation (Figure 7B). Dnmt3a loss eliminated this competitive advantage, leading to $33.2 \% \pm 8.6 \%$ and $28.7 \% \pm 7.8 \%$ of $\mathrm{PB}$ cells derived from $\mathrm{Dnmt} 3 \mathrm{a}^{-/-}$or $\mathrm{PR}^{+/-} \mathrm{Dnmt} 3 \mathrm{a}^{-/}$ donor BM cells, respectively. To determine whether this reflected a defect in peripheralization rather than a true competitive disadvantage, we sacrificed animals 6 months after transplantation and examined donor-derived cells from each genotype in the BM and spleen (Figure 7C). The competitive advantage of $P R^{+/-}$-derived cells was evident in both of these tissues, with $73.4 \% \pm 6.4 \%$ of BM cells and $67.2 \% \pm 3.6 \%$ of splenic cells derived from $P R^{+/-}$-derived donor cells. In contrast, cells derived from $\mathrm{PR}^{+/-} \mathrm{Dnmt} 3 \mathrm{a}^{-/-}$mice comprised only $38.2 \% \pm 12.8 \%$ of total BM cells and $25.9 \% \pm 7.3 \%$ of total splenic cells, with similar competitive deficits observed in the marrow from Dnmt $3 a^{-/}$donors.

DNMT3A loss has been shown to have effects at the level of long-term HSCs $(3,40)$, but $P M L-R A R A$ is primarily expressed in multipotent progenitors (MPPs) and myeloid progenitors in Ctsg-
$P M L-R A R A$ mice (31). We therefore performed additional experiments comparing recipients of $P R^{+/-}$and $P R^{+/-}$Dnmt3a $a^{-/-}$marrow to determine whether DNMT3A loss in this model might negatively affect the ability of hematopoietic stem/progenitor cells (HSPCs) to differentiate into more committed progenitors. We evaluated donor-derived long-term HSCs (as well as more committed progenitor cell populations), and calculated the lineage bias for each genotype, defined as the ratio of donor-derived cells of a given population (e.g., the percentage of long-term HSCs) to the total percentage of donor-derived cells (percentage of Ly5.2+ cells). The expected ratio is $1: 1$ for cells contributing equally to all lineages. Despite an overall competitive disadvantage (compared with $\mathrm{PR}^{+/-}$donor cells), $P \mathrm{R}^{+/-} \mathrm{Dnmt} 3 \mathrm{a}^{-/-}$donor cells were twice as likely to contribute to long-term HSCs (Figure 7D; $P<0.001$, by 2-way ANOVA). In agreement with previous experiments using conditional Dnmt3a/- mice $(3,40)$, the ratio of contributions to other downstream populations (such as short-term HSCs, GMPs, and common myeloid progenitors [CMPs]) was not different among the genotypes (Figure 7E). Collectively, these results suggest that DNMT3A is required for the competitive advantage provided by PML-RARA, perhaps by acting upstream of the myeloid progenitor cells that PML-RARA "reprograms."

To determine whether DNMT3A is required for $P M L-R A R A$ to induce APL in vivo, we transplanted BM from 2- to 2.5-weekold $\mathrm{PR}^{+/-}$or $\mathrm{PR}^{+/-} \mathrm{Dnmt3a} \mathrm{a}^{-/-}$animals into lethally irradiated WT recipients, as described in Figure 2A, and performed a long-term tumor watch. Within 1 year of transplantation, 6 of $16 \mathrm{PR}^{+-}$mice developed the typical features of APL, including splenomegaly, an abnormal proliferation of mature myeloid cells, and atypical promyelocytes in the BM and blood, as previously described (12). In contrast, 0 of $13 \mathrm{PR}^{+/-}$Dnmt3a ${ }^{-/-}$animals developed APL during the same observation period (Figure 7F; $P<0.05$, by both MantelCox and Gehan-Breslow-Wilcoxon tests).

\section{Discussion}

In this report, we have explored mechanisms underlying the mutual exclusivity of AML-initiating fusion genes and mutations in DNMT3A, both of which are common in AML patients. Although retroviral expression of RUNX1-RUNX1T1 or MLL-AF9 induced self-renewal regardless of Dnmt3a status, PML-RARA required DNMT3A to induce self-renewal ex vivo. DNMT3A was likewise required for the self-renewal of myeloid progenitor cells derived from Ctsg-PML-RARA mice and also for their ability to outcompete WT progenitors in a competitive transplantation model. We demonstrated that aberrant self-renewal ex vivo is specifically dependent on the DNA methyltransferase activity of DNMT3A, since neither the DNMT3B gene nor mutant DNMT3A genes from AML patients that are deficient in catalytic activity were able to restore myeloid self-renewal. Reintroduction of WT human DNMT3A was able to restore both normal DNA methylation to a canonically hypomethylated locus and aberrant selfrenewal ability. Finally, Ctsg-PML-RARA mice that were deficient for DNMT3A had a reduced penetrance of APL in vivo.

$P M L-R A R A$ confers an aberrant self-renewal activity to myeloid progenitors, a finding that is evident long before the development of overt leukemia $(31,39,41)$. This property allows myeloid progenitor cells to be serially replated ex vivo and to outcompete 
WT progenitors in competitive transplants $(31,39)$. Aberrant self-renewal may be an essential feature of AML pathogenesis; this phenotype may increase the likelihood that additional, cooperating mutations will occur in cells with increased self-renewal potential. Our findings demonstrate that functional Dnmt3a is required for both the in vivo and ex vivo myeloid self-renewal phenotypes induced by $P M L-R A R A$.

The hematopoietic phenotype we demonstrate for $P R^{+/-}$ Dnmt3a $a^{-/-}$marrow displayed both similarities to and differences from the previously reported phenotypes in serially transplanted hematopoietic cells derived from conditional Dnmt3a-deficient mice. Like Challen et al. (3), we observed a differentiation bias toward self-renewal in Dnmt $3 a^{-/-}$long-term HSCs. However, we found that loss of Dnmt3a decreased the competitive engraftment ability of progenitors derived from the marrow of $\mathrm{PR}^{+-}$mice. We used whole BM in our study, because it was designed to recapitulate our previous studies (31) that demonstrated that whole BM cells from $\mathrm{PR}^{+/-}$mice display a competitive advantage in vivo. There are numerous methodological differences between the two studies, including the use of unfractionated marrow and a single transplantation event in our studies versus the use of sorted longterm stem cells and serial transplantations in the previous study, which may partially explain the apparent discrepancies.

Mayle et al. (40) have shown that sorted HSCs from conditional DNMT3A-deficient mice have an increased propensity to develop both myeloid and lymphoid malignancies after a long period of latency, whereas in this study, DNMT3A deficiency protected Ctsg-PML-RARA mice from developing APL. Further, our $P R^{+/-}$Dnmt3a $a^{-/-}$mice did not spontaneously develop other forms of AML or acute lymphoblastic leukemia (ALL). In this study, we transplanted whole BM rather than purified HSCs, the number of animals at risk was small $(n=13)$, and the study was terminated when significance was achieved at 1 year. It is therefore possible that some of these mice could have developed alternative hematopoietic malignancies had they been followed for a longer period of time. We did not perform an independent tumor watch using the germline Dnmt3a $a^{-/-}$mice, so we do not yet know whether there are biologically relevant differences between these two different DNMT3A-deficient models, or whether differences in phenotypes result from the methodological differences described above. Additional studies will be required to better understand the differences between these model systems.

Dnmt3a was not required for the aberrant ex vivo selfrenewal induced by two common AML-initiating fusions, RUNX1RUNX1T1 and MLL-AF9, and was not required for MLL-AF9 to induce AML in vivo. These data strongly suggest that DNMT3A loss does not cause a general state of hematopoietic dysfunction that is incompatible with the development of AML. In fact, previous studies have shown that Dnmt1 (but not Dnmt3a or Dnmt3b) is highly expressed in a putative leukemic stem cell population from $M L L-A F 9$-expressing AML cells (L-GMPs) $(42,43)$. In this model, haploinsufficiency for Dnmt1 delayed leukemia progression, suggesting that $M L L-A F 9$ requires maintenance methylation by DNMT1 for the induction of leukemia rather than de novo DNA methylation by DNMT3A or DNBMT3B. Likewise, RUNX1RUNX1T1 has been reported to recruit DNMT1 and histone deacetylases in order to silence gene expression, suggesting that it also may require DNMT1, instead of DNMT3A, to induce leukemia in mice $(44,45)$.

We have shown that DNMT3A deficiency attenuates the upregulation of several genes that are normally dysregulated by $P M L-R A R A$ expression in GMP cells. Additional studies will be necessary to determine the specific genes and pathways that $P M L$ RARA uses to drive aberrant self-renewal and how these pathways are affected by DNMT3A. Previous studies have suggested that PML-RARA recruits DNMT3A to induce DNA methylation at specific sites in the genome, resulting in the repression of gene expression $(19,21,24)$. However, alternative hypotheses have also been proposed: a recent study has suggested that PML-RARA binding to DNA may actually protect target sites from CpG methylation; these findings argue that the characteristic methylation changes of APL cells are not actually required for initiation of APL but rather occur after transcription factors leave key binding sites $(46,47)$.

Neither DNMT3B nor catalytically inactive mutants of DNMT3A were capable of restoring replating to $\mathrm{PR}^{+/} \mathrm{Dnmt3a^{-/- }}$ marrow. This specific requirement for Dnmt3a may be due to the fact that the two enzymes have different sequence specificities in vitro $(34,48)$ and have been shown to act on overlapping but distinct genomic regions in vivo $(5,35)$. Therefore, Dnmt3a-specific methylation patterns may be required for $P M L-R A R A$ to induce leukemia.

Collectively, these results suggest an important role for DNMT3A in the development of APL: PML-RARA appears to specifically require DNMT3A to initiate the self-renewal phenotype in myeloid progenitor cells. These findings have helped to define a mechanistic relationship between two recurrent but independent AML driver mutations and add to the growing body of evidence implicating epigenetic changes in the induction of AML.

\section{Methods}

Mice. The Ctsg-PML-RARA and Dnmt $3 a^{-/-}$mice have previously been described $(12,27)$. Both strains were backcrossed with C57Bl/6 mice for more than 10 generations. Dnmt $3 a^{+/}$mice were obtained from the Mutant Mouse Regional Resource Centers (MMRRC) (strain name B6.129S4-Dnmt3a $a^{\text {tm2Enl }} \mathrm{Mmnc}$ ) repository. $\mathrm{PR}^{+/-}$and $\mathrm{Dnmt3a^{-/- }}$ mice and all control genotypes were produced by intercrossing $\mathrm{PR}^{+/-}$with $\mathrm{Dnmt} 3 \mathrm{a}^{+/-}$mice. Dnmt3 $\mathrm{b}^{f / f l}$ mice on a B6 background (B6.129S4[Cg]-DNMT3Btm5.1Enl/Mmnc) were obtained from the MMRRC at the University of North Carolina (UNC). Vav1-Cre mice of the B6 strain were obtained from The Jackson Laboratory (B6. CG-TG[VAV1-CRE]A2KIO/J). Whenever possible, littermate controls were used for all experiments.

$B M$ harvest and transplantation. BM was harvested from the femurs, tibiae, pelvises, and humeri of 2- to 2.5-week-old mice. After lysis of rbc (with ammonium chloride potassium [ACK] buffer: $0.15 \mathrm{M}$ $\mathrm{NH} 4 \mathrm{Cl}, 10 \mathrm{mM}$ KHCO3, $0.1 \mathrm{mM}$ Na2EDTA), cells were washed with FACS buffer, filtered through 50- $\mu \mathrm{m}$ cell strainers (Partec; Sysmex), and resuspended in PBS at 1 million cells $/ 100 \mu \mathrm{l}$ for transplantation. For competitive transplantation experiments, BM was mixed 50:50 with freshly harvested cells from 6-week-old Ly5.1 mice (The Jackson Laboratory). Transplantation was performed by retro-orbital injection of $1 \times 10^{6}$ total BM cells into lethally irradiated Ly5.2 or Ly5.1x5.2 recipients that had received 2 split doses of 550 cGy total body irradiation spaced at 4-hour intervals (Mark 1 Cesium-137 irradiator; JL Shepherd) 24 hours before transplantation. 
Mouse analysis and tumor watch. $\mathrm{PB}$ counts were assessed at regular intervals as indicated by an automated complete blood count (Hemavet 950; Drew Scientific Group). For long-term tumor watch experiments, BM transplant recipients were monitored daily, and animals displaying signs of illness (lethargy, hunched posture, ruffled fur, dyspnea, or pallor) were euthanized, and the spleen and BM were harvested for analysis. Diagnosis of leukemia was made by light microscopic examination of splenic and/or PB cells according to the Bethesda criteria (49) and previously published phenotyping of the Ctsg-PML-RARA and MLL-AF9 viral transduction mouse models. Cytospin tissue slides were stained with Wright-Giemsa stain (SigmaAldrich) and were imaged using a Nikon MICROPHOT-SA microscope equipped with an oil-immersion $50 \times / 0.90$ or $100 \times / 1.30$ objective lens. The tumor watch was terminated 1 year after transplantation.

MSCV vectors. Full-length human DNMT3A (NM_175629) and DNMT3B (NM_006892) cDNAs (Origene) were cloned into MSCVIRES-YFP or MSCV IRES-GFP (Addgene) using PCR-introduced EcoRI restriction digest sites and standard ligation techniques. DNMT3A R882H and Q615* mutations were introduced using a QuikChange II XL mutagenesis kit (Agilent Technologies). PML-RARA cDNA was prepared from the BCR3 PML-RARA cDNA used to create the Ctsg-PML-RARA mouse and cloned into MSCV-IRES-YFP as described above. MSCV MLL-AF9-IRES-GFP and MSCV RUNX1RUNX1T1-IRES-GFP vectors were gifts of Andy Lane (Dana Farber Cancer Institute, Boston, Massachusetts, USA). Virus was produced using cotransfection of MSCV vector and an EcoPak packaging plasmid (Addgene) into 293T cells with standard calcium-phosphate transfection, then harvested 48 hours after transfection and stored at $-80^{\circ} \mathrm{C}$. For transduction of mouse $\mathrm{BM}$, marrow was harvested as above and plated overnight in transplant media containing RPMI supplemented with $15 \% \mathrm{FBS}, 100 \mathrm{ng} / \mathrm{ml}$ stem cell factor (SCF), $10 \mathrm{ng} / \mathrm{ml}$ IL-3, $50 \mathrm{ng} / \mathrm{ml} \mathrm{FLT-3,} \mathrm{and} 10 \mathrm{ng} / \mathrm{ml}$ thrombopoietin (TPO). All cytokines were purchased from PeproTech. Cells were transduced by spinfection at $1,295 \mathrm{~g}$ for 90 minutes at $30^{\circ} \mathrm{C}$ in transplant media with the addition of $1 \mathrm{M}$ HEPES buffer and $0.85 \%$ sodium chloride supplemented with $10 \mu \mathrm{g} / \mathrm{ml}$ polybrene (AmericanBio). After two rounds of spinfection, $\mathrm{GFP}^{+}$or $\mathrm{YFP}^{+}$cells were sorted using an iCyt Synergy II cell sorter (iCyt Mission Technology) and plated in methocellulose for colony formation assays.

Methylcellulose colony formation assay. Cells were plated in triplicate (10,000/plate) in M3534 MethoCult media (STEMCELL Technologies) containing IL-3, IL-6, and SCF (STEMCELL Technologies) and incubated at $37^{\circ} \mathrm{C}$ for 1 week. Each week, clusters of cells meeting the morphologic criteria for CFU granulocyte, erythroid, monocyte, megakaryocyte (CFU-GEMM), CFU granulocyte, macrophage (CFU-GM), CFU granulocyte (CFU-G), or CFU macrophage (CFU-M) (http://www. stemcell.com/ /media/Technical\%20Resources/8/3/E/9/0/28405_ methocult\%20M.pdf?la=en) were counted as myeloid colonies, and cells were lifted using warm DMEM media containing 2\% FBS, spun down, and replated as before. An aliquot of cells was taken for analysis of myeloid markers by flow cytometry.

Methylation capture sequencing. Whole BM was isolated from 2- to 2.5-week-old mice ( $n=3$ per genotype), transplanted into lethally irradiated WT mice, and allowed to stably engraft for 8 to 10 weeks before whole marrow was harvested and DNA isolated using a QiaAmp DNA Mini Kit (QIAGEN). Input DNA $(12 \mu \mathrm{g})$ from each sample was processed with the SureSelectXT Methyl-Seq Target Enrichment System for the Illumina Multiplexed Sequencing protocol (Agilent Technologies). DNA was subsequently fragmented using a Covaris S2 with the following settings: duty cycle: $10 \%$; intensity: 5 ; cycles per burst: 200 ; cycles: 6; and time/cycle: 6 seconds. Library preparation followed the SureSelect Methyl-Seq Library Prep Kit (Agilent Technologies), with minor modifications. After end repair, the DNA was purified using an AMPure XP kit (Beckman Coulter) and recovered in $80 \mu \mathrm{l} 10 \mathrm{mM}$ Tris$\mathrm{HCl}, \mathrm{pH}$ 7.8. Samples were subsequently divided into four A-tailing and adapter ligation reactions following the manufacturer's recommendations for $3 \mu \mathrm{g}$ of input DNA. After ligation, all four reactions for each sample were pooled and processed for hybridization using the SureSelect Methyl-Seq Target Enrichment and Methyl-Seq Hybridization Kits (Agilent Technologies). In the hybridization reactions, we combined the SureSelect Indexing Block \#1 and \#2 oligonucleotides with $5 \mu \mathrm{g}$ Mouse $\mathrm{C}_{\mathrm{o}} \mathrm{t}-1$ DNA (Invitrogen). The hybridized probe/ library fragment duplexes were immobilized with streptavidin-bound paramagnetic M280 particles (Invitrogen). The hybridized (captured) single-stranded DNA (ssDNA) library fragments were eluted from their duplexes and neutralized using the supplied elution and neutralization buffers, respectively. The methyl-captured fragments were bisulfite converted using the DNA Methylation Gold Kit (Zymo Research), and the converted ssDNA library fragments were amplified for 8 cycles with nonindexed primers as described in the manufacturer's protocol. A subsequent amplification incorporated library-specific index sequences during the PCR. The final library amplifications were treated with a 0.8:1.0 ratio for AmpureXP beads/DNA, eluted with $20 \mu \mathrm{l} 10 \mathrm{mM}$ Tris- $\mathrm{HCl}$ (pH 7.8), quantified by Qubit (Invitrogen), and sized with the DNA 1000 Chip Kit (Agilent Technologies) for a molarity determination. All four samples were diluted to $5 \mathrm{nM}$, assayed by qPCR, and, on the basis of qPCR results, diluted to a 2-nM solution. Each 2-nM sample was mixed at equal volumes, and the 2-nM pool was sequenced, $2 \times 100 \mathrm{bp}$, across four lanes of an Illumina HS2000 flow cell using V3 chemistry. BSMAP version 1.037 was used to align the bisulfite sequencing reads. Methylation ratios per base were calculated via the methratio.py script. The methylation ratios were read into a methylKit package in R to generate coverage and methylation statistics and sample correlation, clustering, and differential methylation analysis. The data sets for the CpG capture experiments are deposited in the Short Read Archive (accession number PRJNA283621); the Superseries ID that links the methylation and expression data for this study is Umbrella BioProject PRJNA284138.

Expression profiling. Expression array profiling was performed exactly as described previously (31). Labeled RNA targets were hybridized to Mouse Exon 1.0 ST arrays (Affymetrix), washed, stained, and scanned using standard protocols from the Siteman Cancer Center Molecular Genomic Analysis (MGA) Core Facility (http://pathology. wustl.edu/research/cores/lcg/index.php).

Affymetrix CEL files were imported into Partek Genomics Suite 6.6 (Partek Inc.). Probe-level data were preprocessed, including background correction, normalization, and summarization, using robust multiarray average (RMA) analysis. RMA adjusts for background noise on each array using only the perfect match (PM) probe intensities and subsequently normalizes data across all arrays using quantile normalization $(50,51)$, followed by median polish summarization to generate a single measure of expression (51). Data were filtered to include only core probe sets having a raw expression signal greater than 100 in all samples in order to limit the analysis within well-annotated exons. 
The ANOVA and multi-test correction for $P$ values in the Partek Genomics Suite were used to identify differentially expressed genes. Sample genotypes (WT, $\mathrm{PR}^{+-}, \mathrm{PR}^{+/-} \mathrm{Dnmt} 3 \mathrm{a}^{-/}$, and $\mathrm{Dnmt} 3 \mathrm{a}^{-/-}$) were chosen as the candidate variables in the ANOVA model to obtain genotype-specific expression changes. ANOVA $P$ values were corrected using the Bonferroni method. The list of genes with significant variation in expression levels was generated on the basis of a fold change of 2 and a 0.05 FDR criterion as a significant cutoff.

Exon array data for all samples used in this study have been deposited in the NCBI's Gene Expression Omnibus (GEO) database (GEO GSE68844).

HpaII qPCR assay. DNA was isolated from MethoCult-plated cells after 1 week in MethoCult M3534 media, and genomic DNA (gDNA) was isolated using a QIAmp DNA Mini Kit (QIAGEN). gDNA (500 ng) was digested with $0.5 \mu \mathrm{l}$ (5 units) HpaII enzyme (New England BioLabs) or mock (water) for 90 minutes at $37^{\circ} \mathrm{C}$, then heat inactivated at $80^{\circ} \mathrm{C}$ for 20 minutes. For each 30-ml digest (25 ng gDNA), $1.5 \mu$ l was amplified using a KAPA SYBR FAST qPCR Kit (Kap Biosystems) and PCR primers (IDT). Four amplified regions were selected on the basis of our methylation capture sequencing showing hypomethylation in Dnmt3a-/- versus WT cells, and approximately 200-bp amplicons were designed containing HpaII recognition sites (CCGG) using Primer3 software. Each sample was assayed in triplicate. The percentage of methylation was calculated at $2^{-\Delta \mathrm{Ct}}$, where the $\Delta \mathrm{Ct}$ was $\mathrm{Ct}(\mathrm{HpaII}$ digested) - $\mathrm{Ct}$ (undigested).

Western blot analysis. For blotting of whole embryos, E18 embryos were snap frozen in liquid nitrogen and homogenized with mortar and pestle before lysis in RIPA buffer (50 mM Tris, pH 8.0, $150 \mathrm{mM} \mathrm{NaCl}$, $0.1 \%$ SDS, $0.5 \%$ sodium deoxycholate, $1 \%$ NP-40) supplemented with protease inhibitor cocktail (Sigma-Aldrich). For blotting of retrovirustransduced BM cells, 250,000 cells were rinsed with PBS and resuspended in urea lysis buffer (7 M urea, $2 \mathrm{M}$ thiourea, $30 \mathrm{mM}$ Tris, $\mathrm{pH}$ 8.5) supplemented with protease inhibitor cocktail and then snap frozen in liquid nitrogen. Lysates were boiled in 6X SDS loading dye (0.01 M Tris$\mathrm{HCl}, \mathrm{pH} 6.8,8 \%$ glycerol, $0.1 \mathrm{mg} / \mathrm{ml}$ bromophenol blue, 2\% SDS, $1 \%$ $\beta$-mercaptoethanol) for 5 minutes at $95^{\circ} \mathrm{C}$. Lysates were separated on a $10 \%$ SDS-PAGE gel and transferred onto Hybond-C Extra nitrocellulose membrane (Amersham, GE Healthcare). The membrane was probed with anti-DNMT3A Ab (1:2,000; Cell Signaling Technology; catalog 2160); anti-DNMT3B Ab (1:2,000, Santa Cruz Biotechnology Inc.; catalog sc-10236); or anti- $\alpha$-actin Ab (1:5,000; EMD Millipore). Immune complexes were revealed by peroxidase-conjugated anti-mouse IgG (1:10,000; GE Healthcare) or conjugated anti-rabbit IgG (1:2,500; GE Healthcare) and Western blots were visualized by chemiluminescence using Bio-Rad Clarity Western ECL and either MidSci Classic Blue Autoradiograph Film BX or a ThermoFisher myECL Imager.

Cell staining and flow cytometry. After ACK lysis of rbc, PB, BM, or spleen cells were treated with anti-mouse CD16/32 (clone 93; eBio- science) and stained with the indicated combinations of the following Abs (all were from eBioscience unless otherwise indicated; see Supplemental Table 6): CD34 FITC (clone RAM34); CD11b PE or APC-e780 (clone M1/70); c-Kit PerCP-Cy5.5 or APC-e780 (clone 2B8); CD115 APC or PE (clone AFS98); Gr-1 Pacific Blue (Invitrogen; catalog RM3028); Gr-1 biotin (clone RB6-8C5); B220 PE, APC, or biotin (clone RA3-6B2); CD3 e450 or PE (clone 145-2C11); CD71 PE (clone R17217); Ter-119 Pacific Blue (clone TER-119); CD16/32 APC (clone 93); and FLK2 APC (clone A2F10). Analysis was performed using a FACScan (Beckman Coulter) or an iCyt Synergy II sorter, and data were analyzed using FlowJo, Excel (Microsoft), and Prism 5 (Graphpad Software) software.

Statistics. All statistical comparisons were made using Prism 5 software, except for statistics on sequencing data, which were calculated using $\mathrm{R}$ statistical programming software as described above. The statistical tests used and significance cutoffs are detailed in each figure legend. When used, Student's $t$ test was 2-tailed, and a $P$ value of less than 0.05 was considered statistically significant. All data represent the mean \pm SEM unless otherwise indicated.

Study approval. All mouse experiments were performed in accordance with institutional guidelines and current NIH policies and were approved by the Animal Studies Committee of Washington University.

\section{Author contributions}

CBC, AMV, ERL, DARG, and TJL designed the experiments. CBC, AMV, ERL, and DARG conducted the experiments. CBC, AMV, ERL, TLL, RTD, and VM acquired the data. CBC, SK, and ERL analyzed the data. CBC and TJL wrote the manuscript.

\section{Acknowledgments}

This work was supported by NIH grants CA083962, CA101937, CA162086, and CA197561 and by the Barnes-Jewish Hospital Foundation grant 00335-0505-02 (to T.J. Ley). David Spencer provided invaluable support and advice for several computational aspects of the paper. The Siteman Cancer Center High-Speed Cell Sorter Core was of invaluable assistance and is supported in part by National Cancer Institute (NCI) Cancer Center Support Grant P30 CA91842. The Microarray Core is supported by National Center for Research Resources grant UL1 RR024992.

Address correspondence to: Timothy J. Ley, Washington University School of Medicine, 660 South Euclid Avenue, Campus Box 8007, St. Louis, Missouri 63110, USA. Phone: 314.362.8831; E-mail: timley@wustl.edu.

Tamara L. Lamprecht's present address is: St. Jude Children's Research Hospital, Memphis, Tennessee, USA.
1. Cancer Genome Atlas Research Network. Genomic and epigenomic landscapes of adult de novo acute myeloid leukemia. $N$ Engl J Med. 2013;368(22):2059-2074.

2. Ley T, Ding L, Walter M. DNMT3A mutations in acute myeloid leukemia. $N$ Engl J Med. 2010; 363(25):2424-2433.

3. Challen GA, et al. Dnmt3a is essential for hematopoietic stem cell differentiation. Nat Genet. 2012;44(1):23-31.
4. Yang L, Rau R, Goodell MA. DNMT3A in haematological malignancies. Nat Rev Cancer. 2015;15(3):152-165.

5. Challen GA, et al. Dnmt3a and Dnmt3b have overlapping and distinct functions in hematopoietic stem cells. Cell Stem Cell. 2014;15(3):350-364.

6. Russler-Germain DA, et al. The R882H DNMT3A mutation associated with AML dominantly inhibits wild-type DNMT3A by blocking its ability to form active tetramers. Cancer Cell.
2014;25(4):442-454.

7. Kim SJ, Zhao H, Hardikar S, Singh AK, Goodell MA, Chen T. A DNMT3A mutation common in AML exhibits dominant-negative effects in murine ES cells. Blood. 2013;122(25):4086-4089.

8. Grisolano JL, Wesselschmidt RL, Pelicci PG, Ley TJ. Altered myeloid development and acute leukemia in transgenic mice expressing PMLRAR $\alpha$ under control of cathepsin $G$ regulatory sequences. Blood. 1997;89(2):376-387. 
9. Brown D, et al. A PML-RAR $\alpha$ transgene initiates murine acute promyelocytic leukemia. Proc Natl Acad Sci U S A. 1997;94(6):2551-2556.

10. He LZ, et al. Acute leukemia with promyelocytic features in PML/RAR $\alpha$ transgenic mice. Proc Natl Acad Sci U S A. 1997;94(10):5302-5307.

11. Kogan SC. Mouse models of acute promyelocytic leukemia. Curr Top Microbiol Immunol. 2007;313:3-29.

12. Westervelt $P$, et al. High-penetrance mouse model of acute promyelocytic leukemia with very low levels of PML-RAR $\alpha$ expression. Blood. 2003;102(5):1857-1865.

13. Minucci S, et al. PML-RAR induces promyelocytic leukemias with high efficiency following retroviral gene transfer into purified murine hematopoietic progenitors. Blood. 2002;100(8):2989-2995.

14. Kamashev D, Vitoux D, De Thé H. PMLRARA-RXR oligomers mediate retinoid and rexinoid/cAMP cross-talk in acute promyelocytic leukemia cell differentiation. JExp Med. 2004;199(8):1163-1174.

15. Subramanyam D, et al. PML-RAR $\alpha$ and Dnmt3a1 cooperate in vivo to promote acute promyelocytic leukemia. Cancer Res. 2010;70(21):8792-8801.

16. Hong SH, David G, Wong CW, Dejean A, Privalsky ML. SMRT corepressor interacts with PLZF and with the PML-retinoic acid receptor alpha (RAR $\alpha)$ and PLZF-RAR $\alpha$ oncoproteins associated with acute promyelocytic leukemia. Proc Natl Acad Sci U S A. 1997;94(17):9028-9033.

17. Villa $\mathrm{R}$, et al. The methyl-CpG binding protein $M B D 1$ is required for PML-RAR $\alpha$ function. Proc Natl Acad Sci U S A. 2006;103(5):1400-1405.

18. Lin RJ, Nagy L, Inoue S, Shao W, Miller WH, Evans RM. Role of the histone deacetylase complex in acute promyelocytic leukaemia. Nature. 1998;391(6669):811-814.

19. Fazi F, et al. Retinoic acid targets DNA-methyltransferases and histone deacetylases during APL blast differentiation in vitro and in vivo. Oncogene. 2005;24(11):1820-1830.

20. Datta J, et al. Physical and functional interaction of DNA methyltransferase 3A with Mbd3 and Brg1 in mouse lymphosarcoma cells. Cancer Res. 2005;65(23):10891-10900.

21. Morey L, et al. MBD3, a component of the NuRD complex, facilitates chromatin alteration and deposition of epigenetic marks. Mol Cell Biol. 2008;28(19):5912-5923.

22. Boukarabila $\mathrm{H}$, et al. The PRC1 Polycomb group complex interacts with PLZF/RARA to mediate leukemic transformation. Genes Dev. 2009;23(10):1195-1206.

23. Villa R, et al. Role of the polycomb repressive complex 2 in acute promyelocytic leukemia. Cancer Cell. 2007;11(6):513-525.

24. Di Croce L, et al. Methyltransferase recruitment and DNA hypermethylation of target promoters by an oncogenic transcription factor. Science. 2002;295(5557):1079-1082.

25. Martens JH, et al. PML-RAR $\alpha / R X R$ alters the epigenetic landscape in acute promyelocytic leukemia. Cancer Cell. 2010;17(2):173-185.

26. Wang K, et al. PML/RAR $\alpha$ targets promoter regions containing PU.1 consensus and RARE half sites in acute promyelocytic leukemia. Cancer Cell. 2010;17(2):186-197.

27. Okano M, Bell DW, Haber DA, Li E. DNA methyltransferases Dnmt3a and Dnmt3b are essential for de novo methylation and mammalian development. Cell.1999;99(3):247-257.

28. Jeong $\mathrm{M}$, et al. Large conserved domains of low DNA methylation maintained by Dnmt3a. Nat Genet. 2014;46(1):17-23.

29. Steffen B, et al. AML1/ETO induces self-renewa in hematopoietic progenitor cells via the Groucho-related amino-terminal AES protein. Blood. 2011;117(16):4328-4337.

30. Chen W, et al. Malignant transformation initiated by Mll-AF9: gene dosage and critical target cells. Cancer Cell. 2008;13(5):432-440.

31. Wartman LD, et al. Expression and function of PML-RARA in the hematopoietic progenitor cells of Ctsg-PML-RARA mice. PLoS One. 2012;7(10):e46529.

32. Jurkowska RZ, Jurkowski TP, Jeltsch A. Structure and function of mammalian DNA methyltransferases. Chembiochem. 2011;12(2):206-222.

33. Li H, Rauch T, Chen ZX, Szabó PE, Riggs AD, Pfeifer GP. The histone methyltransferase SETDB1 and the DNA methyltransferase DNMT3A interact directly and localize to promoters silenced in cancer cells. J Biol Chem 2006;281(28):19489-19500.

34. Wienholz BL, Kareta MS, Moarefi AH, Gordon CA, Ginno PA, Chédin F. DNMT3L modulates significant and distinct flanking sequence preference for DNA methylation by DNMT3A and DNMT3B in vivo. PLoS Genet. 2010;6(9):e1001106

35. Jin B, et al. Linking DNA methyltransferases to epigenetic marks and nucleosome structure genome-wide in human tumor cells. Cell Rep. 2012;2(5):1411-1424.

36. Dodge JE, et al. Inactivation of Dnmt3b in mouse embryonic fibroblasts results in DNA hypomethylation, chromosomal instability, and spontaneous immortalization. J Biol Chem. 2005;280(18):17986-17991.
37. Georgiades P, et al. VavCre transgenic mice: a tool for mutagenesis in hematopoietic and endothelial lineages. Genesis. 2002;34(4):251-256.

38. Dodge JE, et al. Inactivation of Dnmt3b in mouse embryonic fibroblasts results in DNA hypomethylation, chromosomal instability, and spontaneous immortalization. J Biol Chem. 2005; 280(18):17986-17991.

39. Welch JS, Yuan W, Ley TJ. PML-RARA can increase hematopoietic self-renewal without causing a myeloproliferative disease in mice. JClin Invest. 2011;121(4):1636-1645.

40. Mayle A, et al. Dnmt3a loss predisposes murine hematopoietic stem cells to malignant transformation. Blood. 2015;125(4):629-638.

41. Wojiski S, et al. PML-RAR $\alpha$ initiates leukemia by conferring properties of self-renewal to committed promyelocytic progenitors. Leukemia 2009;23(8):1462-1471.

42. Trowbridge JJ, Snow JW, Kim J, Orkin SH. DNA methyltransferase 1 is essential for and uniquely regulates hematopoietic stem and progenitor cells. Cell Stem Cell. 2009;5(4):442-449.

43. Trowbridge JJ, Sinha AU, Zhu N, Li M, Armstrong SA, Orkin SH. Haploinsufficiency of Dnmt1 impairs leukemia stem cell function through derepression of bivalent chromatin domains. Genes Dev. 2012;26(4):344-349.

44. Liu S, et al. Interplay of RUNX1/MTG8 and DNA methyltransferase 1 in acute myeloid leukemia. Cancer Res. 2005;65(4):1277-1284.

45. Lasa A, et al. MEIS 1 expression is downregulated through promoter hypermethylation in AML1-ETO acute myeloid leukemias. Leukemia. 2004;18(7):1231-1237.

46. Schoofs T, et al. DNA methylation changes are a late event in acute promyelocytic leukemia and coincide with loss of transcription factor binding. Blood. 2013;121(1):178-187.

47. Rohde C, Schoofs T, Müller-Tidow C. The limited contribution of DNA methylation to PML-RAR $\alpha$ induced leukemia. Oncotarget. 2013;4(1):5-6.

48. Oka M, Rodić N, Graddy J, Chang LJ, Terada N. CpG sites preferentially methylated by Dnmt3a in vivo. J Biol Chem. 2006;281(15):9901-9908.

49. Morse HC 3rd, et al. Bethesda proposals for classification of lymphoid neoplasms in mice. Blood 2002;100(1):246-258.

50. Bolstad BM, Irizarry RA, Astrand M, Speed TP. A comparison of normalization methods for high density oligonucleotide array data based on variance and bias. Bioinformatics. 2003;19(2):185-193.

51. Irizarry RA, et al. Exploration, normalization, and summaries of high density oligonucleotide array probe level data. Biostatistics. 2003;4(2):249-264. 